\title{
Modeling collective learning in design
}

\author{
ZHICHAO WU AND ALEX H.B. DUFFY \\ CAD Centre, Department of Design Manufacture and Engineering Management, University of Strathclyde, \\ Glasgow G1 1XJ, United Kingdom
}

(Received October 6, 2003; AccePted September 3, 2004)

\begin{abstract}
In this paper, a model of collective learning in design is developed in the context of team design. It explains that a team design activity uses input knowledge, environmental information, and design goals to produce output knowledge. A collective learning activity uses input knowledge from different agents and produces learned knowledge with the process of knowledge acquisition and transformation between different agents, which may be triggered by learning goals and rationale triggers. Different forms of collective learning were observed with respect to agent interactions, goal(s) of learning, and involvement of an agent. Three types of links between team design and collective learning were identified, namely teleological, rationale, and epistemic. Hypotheses of collective learning are made based upon existing theories and models in design and learning, which were tested using a protocol analysis approach. The model of collective learning in design is derived from the test results. The proposed model can be used as a basis to develop agent-based learning systems in design. In the future, collective learning between design teams, the links between collective learning and creativity, and computational support for collective learning can be investigated.
\end{abstract}

Keywords: Collective Learning; Creativity; Protocol Analysis; Team Design

\section{INTRODUCTION}

The objective of this paper is to present an investigation into the nature of collective learning in design. Such an investigation can provide a basis for the development of agent-based learning systems in design.

Learning is considered as a process of acquisition of new knowledge during which knowledge transformation can occur (Kolb, 1984; Kim, 1993; Marton \& Booth, 1997). Learning is linked with knowledge either at a symbolic or subsymbolic level (Kocabas, 1991). It is difficult and probably impossible to find a definition of knowledge that is universally accepted by all researchers. In this paper, knowledge is concerned with what the nature of the design artifact is, how particular design activities are carried out, and how design activities are organized and executed, derived from Zhang's (1998) work. Collective learning is understood as how a group of agents (either humans or computer systems) interact with and learn from each other in a design context, such as team design.

Reprint requests to: Zhichao Wu, CAD Centre, Department of Design Manufacture and Engineering Management, University of Strathclyde, 75 Montrose St., Glasgow G1 1XJ, UK. E-mail: chao@cad.strath.ac.uk
Within existing works, the understanding of the phenomenon of learning in design is limited (Sim, 2000). Gero and Neill (1998, p. 21) argued the following:

Given the large body of design research it is surprising how little we know about designing: the activity carried out by designers. Design research over the last three decades has largely concentrated on computer-based models of design. This certainly made sense since the computer holds the promise of becoming a tool to aid human designers. ... There still remains a paucity of literature on how designers design which is based not on anecdotes or on personal introspection but on reproducible results, results which are capable of characterizing designing.

Recently, a model of learning in design in the context of one designer was developed that describes how an individual agent learns during the design process and how design and learning are linked with each other (Sim, 2000). Some works are related to collective learning in design, such as those in Duffy and Duffy (1996), Grecu and Brown (1996a, 1996b, 1996c), and Stumpf and McDonnell (2001, 2002). However, currently, the nature of collective learning on human beings has not been revealed. 
A protocol analysis approach is adopted in this paper. Cross et al. (1996) argued that protocol analysis has become "the most likely method (perhaps the only method) to bring out into the open somewhat mysterious cognitive abilities of designers . . . It is difficult to imagine how else we might examine what is going on inside people's heads, other than by asking them to tell us what they are thinking." In essence, protocol analysis relies on the verbal data produced by subjects of their own cognitive activities. The approach is used to test hypotheses of collective learning in design. The model of collective learning in design is derived from the test results.

The content of this paper is organized as follows. Section 2 presents related works. In Section 3, hypotheses of collective learning in design are made, which include those of team design, collective learning, and their interactions. In Section 4, three experiments carried out and used to test the hypotheses of collective learning in design are described. Section 5 presents the test results with the observed examples illustrated in Appendix A. Section 6 presents a model of collective learning in design, derived from the test results. In Section 7, the strengths and the weaknesses of the model and the research approach are discussed, and future avenues are identified. Finally, Section 8 presents the conclusion.

\section{RELATED WORKS}

Within existing literature, different types of learning are reported, such as individual, team, and organizational learning. The types of learning and their relations are described in Figure 1. Team learning involves individual learning, while organizational learning involves both individual and team learning. Collective learning occurs in a team or an organization, resulting in a team or an organization as a whole to learn. Other terms in domains of psychology, engineering design, and distributed artificial intelligence (DAI), such as collaborative learning, shared learning, and agent-based learning, are referred as collective learning in that they represent that agents interact with and learn from each other.
Sim (2000) investigated learning in design in the context of one designer. The elements for a design activity and a learning activity and their links were formalized and observed. The elements for a design activity included design goal, input knowledge, and output knowledge where the design goal guides the design activity, input knowledge is the knowledge sources required to perform the activity, and output knowledge is the result of a design activity. The elements for a learning activity included learning goal, input knowledge, learned knowledge, rationale trigger, and knowledge transformer. The learning goal represents the purpose of carrying out a learning activity. Input knowledge is described as the sources of the knowledge to learn from. Learned knowledge is the output of the learning activity. Rationale triggers represent the reasons that trigger learning. Knowledge transformers are the operators that transform input knowledge to output knowledge (see Table 1), which includes seven opposed pairs, namely, generalization / specialization, similization/dissimilization, association/ disassociation, agglomeration/decomposition, derivation (reformulation)/randomization, explanation/discovery, and characterization/determination. The elements for designing and learning are linked together through designing and learning interactions. The links between them were identified as teleological, rationale, and epistemic. The teleological link is related to the goals, that is, the design goal can precede a learning goal or vice versa. The rationale link is concerned with the reasons that trigger learning, including novelty driven, expedience driven, conflict avoidance, and failure avoidance. The epistemic link is concerned with knowledge acquisition and transformation process during the design and learning process.

Stumpf and McDonell $(2001,2002)$ investigated learning within design teams, based upon the reflective practice framework. The learning styles of designers, their perceptions, and their relationships have been analyzed (Stumpf $\&$ McDonnell, 2001). The interactions between designers with the focus on the process of frame negotiation were also investigated (Stumpf \& McDonnell, 2002). However,

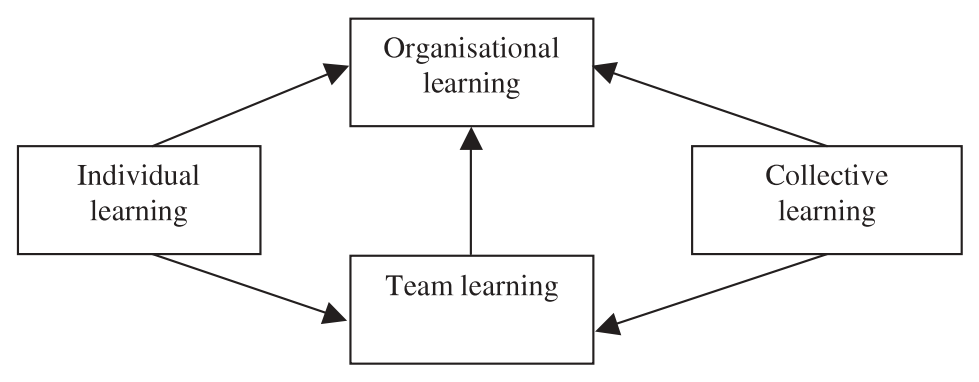

Key:

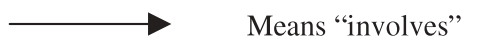

Fig. 1. Types of learning. 
Table 1. Pairs of knowledge transformers

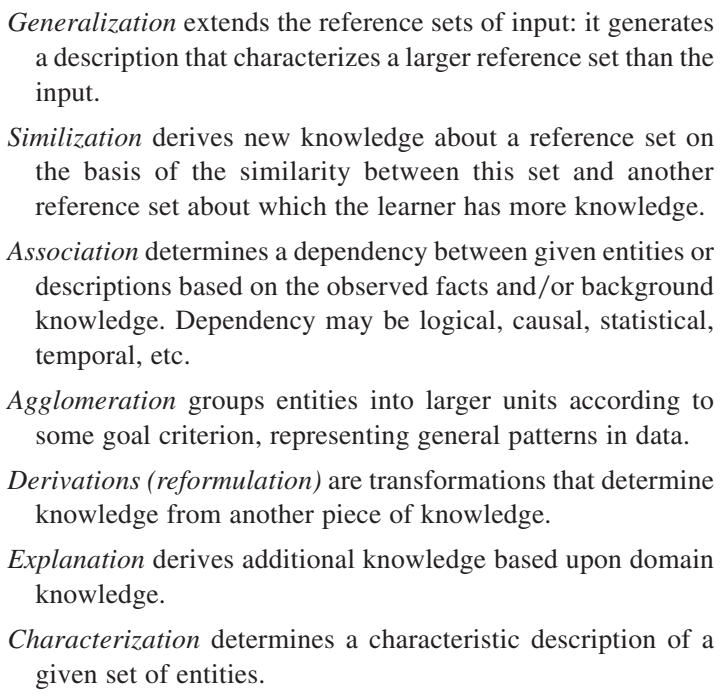

Similization derives new knowledge about a reference set on the basis of the similarity between this set and another reference set about which the learner has more knowledge.

Association determines a dependency between given entities or descriptions based on the observed facts and/or background knowledge. Dependency may be logical, causal, statistical, temporal, etc.

Agglomeration groups entities into larger units according to some goal criterion, representing general patterns in data.

Derivations (reformulation) are transformations that determine knowledge from another piece of knowledge.

Explanation derives additional knowledge based upon domain knowledge.

Characterization determines a characteristic description of a given set of entities.

Specialization narrows the reference set of objects.

Dissimilization derives new knowledge on the basis of the lack of similarity between the compared reference sets.

Disassociation asserts a lack of dependency, determining that a given instance is not an example of some concept is a disassociation transmutation.

Decomposition splits a group (or a structure) of entities of into subgroups according to some goal criterion.

Randomization transforms one knowledge segment into another by making random changes.

Discovery derives new knowledge without underlying domain knowledge.

Discrimination determines a description that discriminates (distinguishes) the given set of entities from another set of entities.

Adapted from Sim (2000).

within their research, the focus is still on individual learning, although in the context of team design.

Duffy and Duffy (1996) developed the concept of Shared Learning. In shared learning, the designer and computing system, acting as a learning assistant, co-learn. That is, a designer learns new knowledge from a learning assistant that automatically learns and presents previously implicit, and therefore unrepresented knowledge. To some degree, shared learning reflects the idea of collective learning in that a designer and a computer co-learn. Grecu and Brown $(1996 a, 1996 c)$ investigated agent-based learning in design using single function agents. Agents can decide autonomously to start a learning process. They can also cooperatively carry out a learning task through sharing learning information and learning results. Their work reflects the perspective of collective learning because agents can collaboratively perform a learning task. However, their research has focused on developing computational support, which can be simplified from design reality.

Within the last few decades, there has been considerable research on team and organizational learning in organizational studies (Argyris \& Schön, 1978; Neergaard, 1994; Cross \& Israelit, 2000). Team learning is oriented toward an effort to improve collaboration, and integrate specialized functional and technical knowledge (Cross \& Israelit, 2000). Organizational learning is understood as the results that members of the organization respond to changes in the internal and external environments of the organization, and thus to change the norms, strategies, and assumptions in the organization (Argyris \& Schön, 1978). The "organization" itself does not learn, but members of the organization, as agents, undertake the task of learning. In the domain of psychology, collaborative learning research was initiated by the educator Edwin Mason (1970). Collaborative learning is concerned with the situation in which two or more people learn something together (Dillenbourg, 1999). "Two or more people" can be interpreted as a school class, a team, or a community. "Learn something" can occur by "follow a course," "study course material," and "perform learning activity in problem solving." The learners can learn "together" with different forms of interactions, such as face to face, and computer mediated.

Within existing literature, there is a paucity of work on collective learning in design on human beings, although considerable research is reported in the domains of psychology and organizational study. A model of learning in design in the context of an individual designer has been developed within which the elements of a design activity and a learning activity were formalized and the interactions between design and learning were identified. The model can provide a basis to investigate collective learning in design. Few works have been carried out on computational supported collective learning in design.

\section{HYPOTHESES}

In this section, the hypotheses of team design, collective learning, and their interactions are made based upon existing theories and models in design and learning.

\subsection{Team design}

A design activity is a goal-directed problem-solving process (Gero, 1990; Fricke, 1996; Sim, 2000), which is char- 


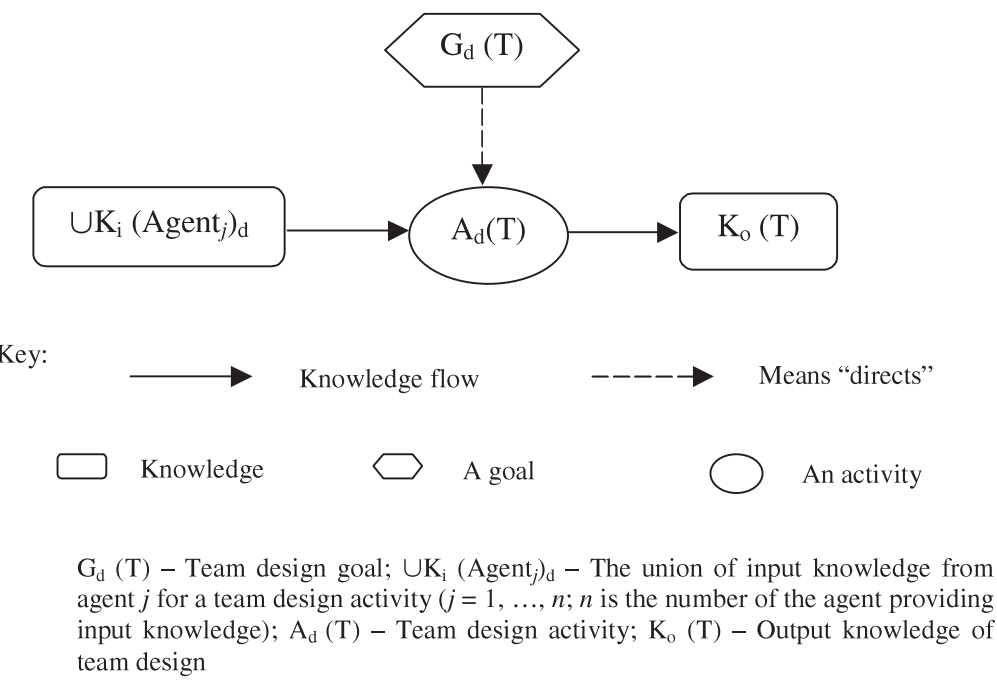

Fig. 2. The elements of a team design activity.

acterized as a change of the states of information by means of a design method or methodology (Hubka \& Eder, 1996). Sim (2000) suggested that designing as a complex cognitive activity can be described at the knowledge level. There is input knowledge for a design activity that is featured as the current states of the design, constraints, and internal and external requirements. Input knowledge enables the design to progress to the design solution, through which output knowledge is created. In a design team, it has been observed that different types (e.g., declarative or procedural) and forms (e.g., concrete or abstract) of knowledge were displayed in the acts of idea presentation, sharing, mutation, and acceptance (Radcliffe, 1996). The following hypothesis is made (see Fig. 2):

Hypothesis 1.1. A team design activity uses input knowledge and the design goal and produces output knowledge. That is, a team design can be modeled with three elements, namely, the team design goal, input knowledge, and output knowledge.

The team design goal directs the team design activity. Input knowledge is represented as a union of input knowledge from individual agents. Output knowledge is produced as the result of an activity. (Note that the focus is on the results of the output of a team, but not on that of individual agents.) Modeling a team design activity in this way provides a basis for the investigation of collective learning in that learning is concerned with some form of knowledge acquisition and transformation.

\subsection{Collective learning}

In this section, the hypotheses about collective learning are made, with regard to the existence, the elements, forms, and the interconnection between collective memory and collective learning.

\subsubsection{The existence of collective learning}

Designing was understood to be linked to learning (Cross \& Nathenson, 1981; Gero, 1990; Duffy \& Duffy, 1996; Sim, 2000). That designs learn during a design process has been observed in the studies of designers since the early 1960s (Marples, 1960; Levin, 1966; Eastman, 1970). Cross and Nathenson (1981) stated that "the design process is often linked to a learning process: in the course of designing, the designer is learning about the problem, its solution, and their relationships." The links between designing and learning were detailed and evaluated by Sim and Duffy within a model of learning in design (Sim, 2000).

Collective learning was reported in related works in psychology and organizational study, although few works are found in the domain of design. Dillenbourg (1999) argued that two or more people could learn something together. The OADI-SMM model describes how individuals learn collectively and results in organizational learning (Kim, 1993).

In team working, agents interact with each other, during which agents share their knowledge and have influence over their decision making (West, 1994), which provides the opportunity for collective learning. As such, Hypothesis 2.1 is made.

Hypothesis 2.1. Collective learning exists in team design.

\subsubsection{Elements of a collective learning activity}

Grecu and Brown $(1996 b, 1998)$ proposed "dimensions" of learning in design that include triggers of learning, elements supporting learning, the types of learned knowledge, availability of knowledge for learning, methods of learning, consequences of learning, and local and global learning. The intention for such a set of dimensions is to provide 


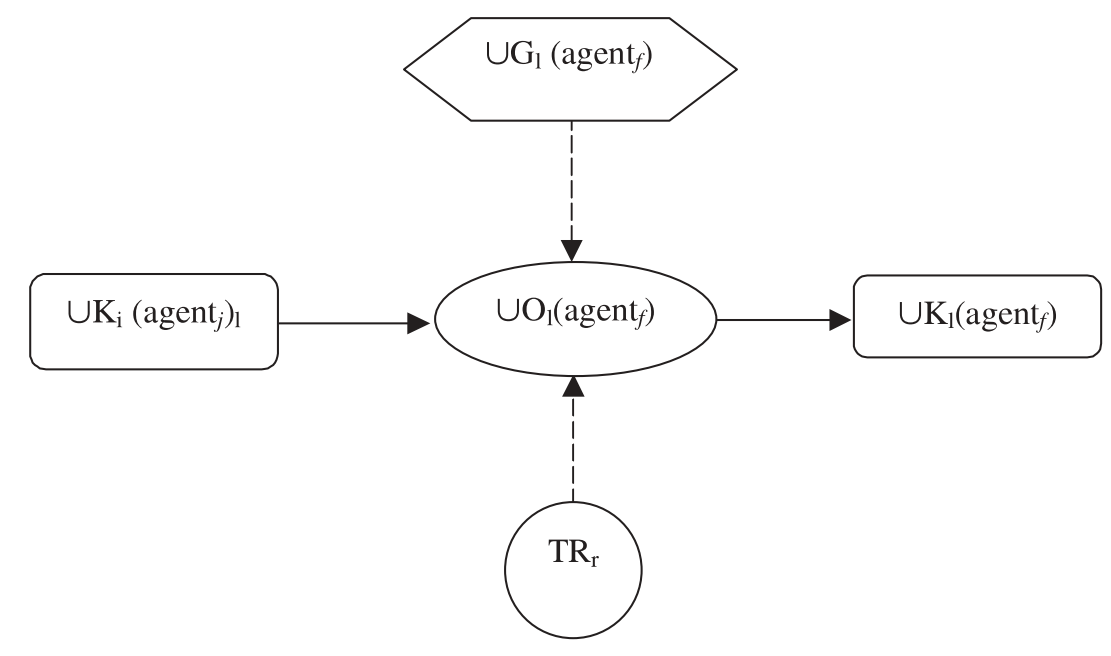

Key:
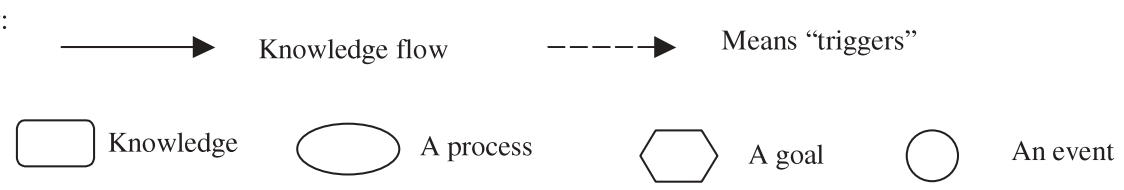

$\cup \mathrm{K}_{\mathrm{i}}\left(\text { agent }_{j}\right)_{1}$ - the union of input knowledge for a learning activity $(j=1, \ldots, n ; n$ is the number of agents who provide the input knowledge); $\mathrm{G}_{1}\left(\right.$ agent $\left._{f}\right)$ - agent $f$ 's learning goal $(f=1, \ldots m ; m$ is the number of agents who perform learning activities); $\mathrm{O}_{1}\left(\right.$ agent $\left._{f}\right)$ - agent $f$ 's learning operator; $\mathrm{K}_{1}\left(\right.$ agent $\left._{f}\right)$ - Learned knowledge of agent $f ; \mathrm{TR}_{\mathrm{r}}-$ rationale trigger.

Fig. 3. The elements of a collective learning activity.

a framework for future exploration in learning. Reich proposed more comprehensive dimensions of learning in design (Reich, 1998), including learners (i.e., who is learning?), finiteness of knowledge (i.e., why does the learner want to learn?), timing (i.e., when does the learner learn and when are the results of learning needed?), activities (i.e., what is the learner doing?), improvements (i.e., what is learned?), prerequisites and processes (i.e., how does the learner learn?), outcome (i.e., what are the consequences?), and cost (i.e., what resources are needed to carry out the learning abilities?).

The elements of a collective learning activity are modeled based upon the dimensions of learning in design and those of an individual learning activity (Sim, 2000). Thus, Hypothesis 2.2 is proposed (see Fig. 3).

HYPOTHESIS 2.2. A collective learning uses input knowledge from different agents and produces learned knowledge with a process of knowledge acquisition and transformation, which is triggered by learning goals and rationale triggers. That is, the elements of a collective learning activity consist of learning goals, input knowledge, learned knowledge, rationale triggers, and learning operators.

Note that a rationale trigger is different from a learning goal in that it represents the reasons that trigger a learning activity rather than its purpose. In addition, the term learning operator replaces the term of knowledge transformer in Sim's model, given the fact that learning can occur without knowledge transformation. The mapping between the dimensions of learning and the collective learning elements is depicted in Table 2.

The elements for a collective learning activity are similar to those for an individual learning activity; however, they have different meanings. The purpose of the learning goal in collective learning is that agents learn collectively. Two agents or more can provide input knowledge for a learning activity. As a result, one agent or more can learn. Rationale triggers are the reasons that trigger agents to learn from each other. The learning operators in collective learning are linked with different agents and reside in the designers who learn.

Persidis and Duffy (1991) postulated that learning occurs in three distinct ways (see Fig. 4):

1. Acquisition: a designer acquires knowledge directly from other designers or knowledge resources.

2. Modification: a process of altering existing knowledge.

3. Generation: new knowledge is created from existing knowledge.

Thus, the learning process can occur without knowledge transformation (i.e., acquisition) or with knowledge trans- 
Table 2. The mapping between the "dimensions" of learning and the elements of a collective learning activity

\begin{tabular}{|c|c|c|}
\hline Reich's Dimension & $\begin{array}{l}\text { Grecu \& Brown's } \\
\text { Dimension }\end{array}$ & $\begin{array}{l}\text { Elements of Collective } \\
\text { Learning Activity }\end{array}$ \\
\hline 1. Who is learning? & Local vs. global learning & Agents in a design team \\
\hline 2. Why does the learner want to learn? & Trigger for learning & Rationale triggers, learning goal \\
\hline 3. When does the learner learn? & - & Rationale triggers and learning goal \\
\hline 4. What is the learner doing? & - & Team design activities \\
\hline 5. What is learned? & What might be learned & Learned knowledge \\
\hline 6. How does the learner learn? & $\begin{array}{l}\text { Elements supporting learning, availability } \\
\text { of knowledge, and methods }\end{array}$ & $\begin{array}{l}\text { Input knowledge, learning operators, and } \\
\text { learned knowledge }\end{array}$ \\
\hline 7 What are the consequences of learning? & Consequences of learning & Learned knowledge \\
\hline $\begin{array}{l}\text { 8. What resources are needed to carry out } \\
\text { the learning activity? }\end{array}$ & - & Input knowledge \\
\hline
\end{tabular}

formation (i.e., modification and generation). (Transformation here represents that of the knowledge itself, not the knowledge states of agents.) Knowledge transformation during the learning process is detailed, with the knowledge transformers identified in Table 1. Thus, it is proposed that there are two types of learning operators in collective learning, that is, knowledge acquisition without or with transformation. In the first type, agents acquire knowledge from others directly. In the second type, agents acquire a piece of knowledge and transform that knowledge into another state with the knowledge transformers used.

Different types of knowledge can be learned. For instance, Grecu and Brown $(1996,1998)$ suggested that the types of learned knowledge include constraints relating parameters or other elements of the design, dependencies between design parameters, support in favor of or against a decision, design rules, methods, and plans, analogical associations, preferences, conditions for rules, actions, and tasks, consequences of design decisions, failures, and conflicts, heuristics for failure recovery and conflict resolution, and successful design and design processes. It was observed that these types of knowledge are learned individually (Sim, 2000). It is assumed that they can also be learned collectively.

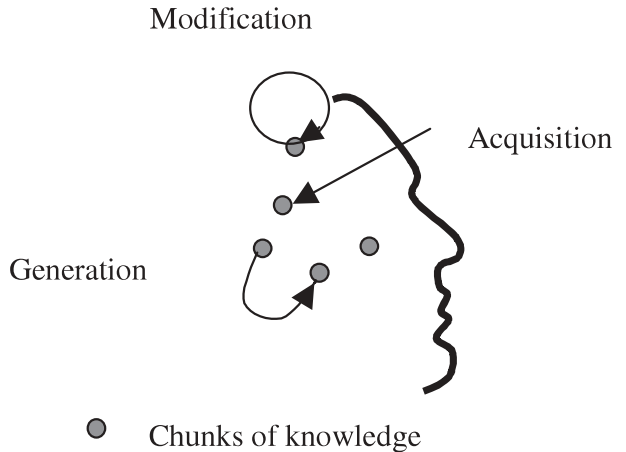

Key: $\quad$ O Chunks of knowledge

Fig. 4. Three types of learning processes (Persidis \& Duffy, 1991).

\subsubsection{Forms of collective learning}

Agents can interact with each other in different forms (e.g., directly or indirectly, and with some or all of others; Hare, 1976). With different forms of agent interactions considered, there can be different forms of collective learning. In addition, Sen and Sekaran (1996) suggested that learning in a multiagent system can be categorized into different forms with respect to the goal of learning, and an agent's involvement. Although the classification is in the context of agent-based computer systems, it triggers the idea that forms of collective learning in team design practice can be categorized in these ways. Therefore, Hypothesis 2.3 is made.

HYPOTHESIS 2.3. There are different forms of collective learning with respect to the forms of agent interactions, the goal of learning, and an agent's involvement.

With respect to the forms of agent interactions, four basic forms of learning may exist: one-one, one-many, manyone, and many-many learning. One agent can learn from another, named one-one learning. One agent can acquire a piece of knowledge from another directly. Many agents can learn from another at the same time or at different times, called one-many learning, and many agents can acquire the same piece of knowledge from the same agent. Conversely, based upon the same knowledge input, different knowledge is generated within many agents using different knowledge transformers. It is argued that each person perceives and interprets the world differently (Kelly, 1955; Gero \& Kannengiesser, 2002). With the same knowledge input, different output can be produced within different agents. One agent can learn from many others, called many-one learning. Other agents can have complementary, different or even conflicting input knowledge. The agent can select, or combine different pieces of knowledge and learned knowledge is produced. Many agents (e.g., a team of designers) can learn from many others (e.g., another team of designers), namely, many-many learning. For example, a team of designers can observe another design team from which they 
could learn. These four forms of collective learning can be combined and other forms can be derived. For example, one-one-many learning is the result of the combination of one-one learning with one-many learning. One agent can learn a piece of knowledge and can share that knowledge with many others, and as a result others can learn that knowledge.

With respect to the goal of learning, there may be two forms of collective learning, namely, assisted, and joint. In form 1, one agent or more assist another or a group of others to learn. The goal is to assist and improve one or some (but not all) of them. In form 2, agents assist one another during the learning process. The goal is to improve all of them.

With respect to an agent's involvement, there may be two additional forms of collective learning: learning in which one is not necessary to be involved, and learning in which one must be involved. In form 3 , the involvement of the agent is not a necessary condition for achieving the pursued learning goal. The agent can be replaced by other agents. In form 4 , the agent cannot be replaced by others, and the learning goal cannot be achieved without the agent. It may be the reason that the agent may have some special expertise that others do not have.

\subsubsection{Collective memory}

Learning and memory are interconnected in that what we already have in our memory affects what we learn and what we learn affects our memory (Schank, 1982; Kim, 1993; Ashcraft, 1994). Memory is understood as a knowledge storing and retrieval process and a storage place (Ashcraft, 1994). Collective memory represents the collection of the memories that can be made available to carry out collective learning and/or team design activity. Such memories can be conflicting, complementary, or the same. Thus, Hypothesis 2.4 is presented.

HYPOTHESIs 2.4. Collective memory and collective learning are interconnected.

What is learned is stored in collective memory, which can be used for current or future design practice or learning activity.

\subsection{Team design and collective learning interactions}

A learning process, described as a cycle of learning and doing, has been reflected in existing learning models in the domains of psychology, organization, and design (Dewey, 1938; Lewin, 1951; Piaget, 1970; Argyris \& Schön, 1978; Kolb, 1984; Kofman, 1992; Kim, 1993; Duffy \& Duffy, 1996). Hypothesis 3.1 is the following:

Hypothesis 3.1. Team design and collective learning are linked with each other and can be described as cyclic in nature.
Collective learning occurs in team design. What is learned collectively can be used for the current and future design practice.

Adapting the three types of links (i.e., teleological, rationale, and epistemic) in individual learning (Sim, 2000), the following hypothesis is proposed:

HYPOTHesis 3.2. The three link types (i.e., teleological, rationale, and epistemic link) also exist in the context of collective learning.

The teleological link is the relationship between the learning goal and the team design goal. The team design goal can precede the learning goal, or vice versa. The rationale link is related to rationale triggers that are concerned with the reasons to trigger collective learning. The epistemic link represents the phenomenon that agents acquire and/or transform knowledge from each other during team design processes, with learning operators linking input knowledge with learned knowledge.

\section{THE EXPERIMENTS}

Three experiments were carried out to test the hypotheses of collective learning, in which team design meetings were recorded using video camera. In the first experiment, a meeting of a student design team was recorded. There were four team members from the fifth (final) year of a Masters of Engineering Product Design course. The team members are represented as GM, PH, MH, and DB in the protocol. The students were tasked with designing a fluid delivery system for a 3-dimensional printer. The design was in its conceptual design stage. Within the meeting, the team designed the lip of the cartridge, means to seal the tube, the size and volume of the tank, the way to replace the tank, and the layout of the printer. During the meeting, the designers used pen and paper to sketch their design ideas. The sketches drawn by the designers were used to assist understanding of the verbal data. The whole design session lasted $1 \mathrm{~h}$ and $13 \mathrm{~min}$. In the second recorded meeting, there were six members, composed of two fifth-year students from the product design course, two fifth-year students from the manufacturing course, the team design project advisor, and a member from the client company. They are represented as KS, NB, MM, CF, AT, and AF in the protocol. They were tasked with redesigning a bitumen tank for road construction. The drawings of the past designs were available for their references. In the meeting, they designed the insulation, the heating element, the layout of the bottom of the tank, and the attachment between the inner and the outer tank. The meeting lasted $1 \mathrm{~h}$ and $3 \mathrm{~min}$. In the third meeting, the same members, except the course advisor (AT) and the client (AF), were involved, working on the bitumen tank design project. In the meeting, they focused on designing the size, the heating pipe, and the sections of the tank, which lasted $1 \mathrm{~h}$ and $21 \mathrm{~min}$. In the recording process, the 
Table 3. The coding scheme

\begin{tabular}{|c|c|c|}
\hline \multicolumn{2}{|c|}{ Elements } & Codes \\
\hline Team design activity & $\begin{array}{l}\text { Agent }_{i} \text { 's input knowledge } \\
\text { Team design goal } \\
\text { Output knowledge }\end{array}$ & $\begin{array}{l}K_{\mathrm{i}}\left(\text { Agent }_{i}\right) \\
G_{\mathrm{d}}(T) \\
K_{\mathrm{o}}(T)\end{array}$ \\
\hline $\begin{array}{l}\text { Collective learning } \\
\text { activity }\end{array}$ & $\begin{array}{l}\text { Agent }_{i} \text { 's input knowledge } \\
\text { Agent }_{i} \text { 's learning goal } \\
\text { Agent }_{i} \text { 's learning operator } \\
\text { Agent }_{i} \text { 's learned knowledge } \\
\text { Agent }_{i} \text { 's rationale trigger }\end{array}$ & $\begin{array}{l}K_{\mathrm{i}}\left(\text { Agent }_{i}\right) \\
G_{1}\left(\text { Agent }_{i}\right) \\
O_{1}\left(\text { Agent }_{i}\right) \\
K_{1}\left(\text { Agent }_{i}\right) \\
\text { TR }_{\mathrm{r}}\left(\text { Agent }_{i}\right)\end{array}$ \\
\hline
\end{tabular}

recorder moved around and recorded both the overall views and the local views. The overall views captured the overall interactions of the designers. The local views captured the gestures and sketching activities of individual designers.

The recorded tapes of the three experiments were transcribed. The coding scheme is derived from the hypotheses made in Section 3, which included elements of a team design activity and a collective learning activity. The elements and their code are presented in Table 3 . The transcribed data are encoded using the coding scheme.

After the data were encoded, interviews of each member involved in the projects were carried out to check the encoded data, aiming to reduce the subjectivity of the protocol analysis approach. During the interviews, the recorded tapes were used to remind the participants what happened during their meetings. Questions were asked such as "Did collective learning occur in this design activity?", "What do you learn in this particular design activity?", and "What triggered you to learn?"

\section{TEST RESULTS}

In this section, the test results of team design, collective learning, and their links are presented with the observed examples illustrated in Appendix A.

\subsection{Team design}

Team design goal was identified in the observed design sessions (see the examples in Section A.1.1 for details). Input knowledge of a team design activity could be from one agent or more (see Examples 1-1 and 1-2; note the examples in Appendix A are referred to as $x-y$ ). Besides from agents, input knowledge can come from external sources (e.g., past design cases), which is defined as External Knowledge (i.e., the knowledge exists outside the agents). In sessions one and two of team two, past design drawings were used as input knowledge for the new design context. The input knowledge from two agents or more can be complementary, alternative, or conflicting (see Examples 1-3, $1-4$, and 1-5). Output knowledge was observed in the meet- ing sessions (see examples in Section A.1.3). A variety of knowledge was produced during the design processes, such as the knowledge about the insulation of the tank, about the layout of the heating elements, about the size of the tank, and about the connection between the inner and the outer tank.

The test results support that design goal and output knowledge exists in team design; however, they partially reject Hypothesis 1.1 on input knowledge in that it includes external knowledge, not only those from agents.

\subsection{Collective learning}

It was observed that collective learning existed in team design (see the examples in Section A.2.1). Collective learning did not occur in all the team design activities. Figure 5 illustrates the occurrences of collective learning throughout the design processes in the meeting sessions. In some time periods, collective learning cannot be identified, because the designers kept silent, or the design activities were carried out and designers verbalized their thoughts but there was no indication of collective learning.

The test results of input knowledge show the following:

1. It could be provided by one agent or more or from other agents plus the agent themselves (see Examples 2-1, 2-2, and 2-3).

2. It could come from other sources (i.e., the diagrams of past designs) besides those from agents (see Example 2-4).

3. It could be the same as that for the design activity, which indicated that in some situations a learning activity and a design activity could not actually be separated (see Example 2-5), or the input knowledge for the collective learning activity could be part of that for the design activity (see Example 2-6).

4. Some learning activities were not linked with a design activity in which there was only input knowledge for a learning activity identified (see Example 2-7).

Types of learned knowledge (see Section A.2.2) were observed. Figure 6 illustrates the types of learned knowledge and their distributions.

Two types of knowledge are identified that can only be learned collectively, namely, common knowledge, and the knowledge of knowledge states of other agents, which is based on either of these two criteria: the learning activities must involve two agents or more or there should be interactions between agents in the learning process. Common knowledge is defined as the knowledge known by all the agents in a team (see Example 2-8). One agent can also learn the knowledge states of others (see Example 2-9). Learned knowledge can be part of or the same as the output knowledge of a team design activity (see Examples 2-10 and 2-11). 

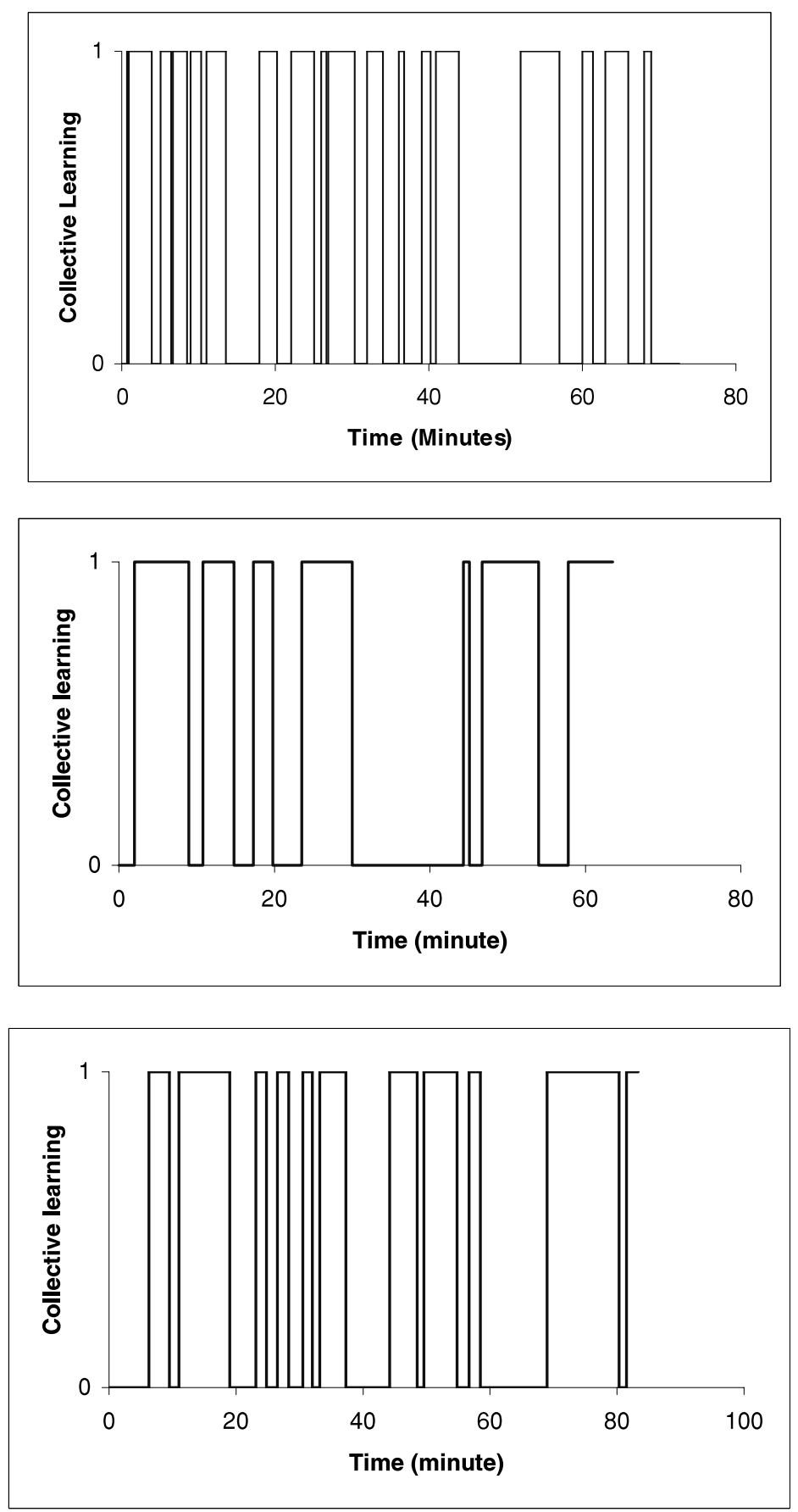

Key: "0" - no collective learning during the time period; " 1 " - the occurrence of collective learning during the time period

Fig. 5. The occurrence of collective learning in team one and meeting sessions one and two of team two.

Learning goals were observed in the meeting sessions (see examples in Section A.2.2).

Rationale triggers were identified as explanation from other agents (Example 2-12), confirmation from other agents (Example 2-13), agreement between agents (Example 2-14), disagreement between agents (see Example 2-15), failed design experience (Example 2-16), and successful design experience (Example 2-17). However, not all the learning activities in the observed team meeting had rationale triggers. There were $35 \%$ of the learning activities in team one 

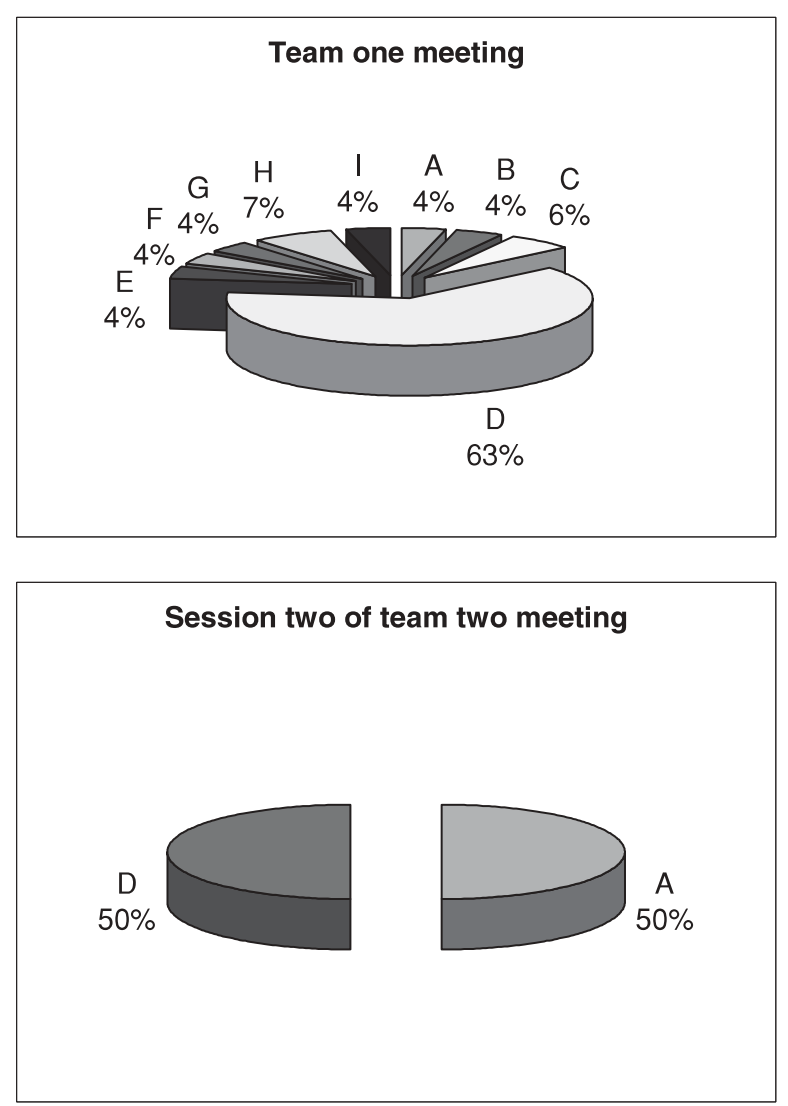

\section{Session one of team two meeting}

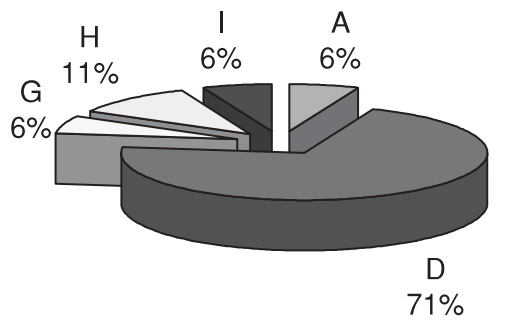

Fig. 6. The distributions of types of learned knowledge.

meeting and $29 \%$ of the learning activities in session one and $42 \%$ in session two of team two meeting without rationale triggers identified. Agents could learn from others only because the knowledge was present in their meetings.

The two types of learning operators (i.e., knowledge acquisition without or with transformation) were observed. There are $51 \%$ of learning activities without knowledge transformation in the meeting session in team one, $71 \%$ in meeting session one, and $73 \%$ in meeting session two of team two. An example of the first type of learning operator is illustrated in Example 2-18. In the second type of learning operator, the knowledge transformers identified included discovery (Example 2-19), derivation (Example 2-20), and specialization (Example 2-21).

The observed examples thus partially reject Hypothesis 2.2 in that input knowledge includes not only those from agents but also those from the environment and that the learning goals and rationale triggers do not exist in all learning activities.

With respect to forms of agent interactions, the four basic forms of learning, namely, one-one (Example 2-22), onemany (Example 2-24), many-one (Example 2-26), and many-many (Example 2-27), were identified. The derived forms, such as one-one-many, were also observed (Example 2-28). One-one learning can result in mutual learning, in which knowledge states of agents mutually evolve (Exam- ple 2-23). The concept of mutual learning is explained in Figure 7. Agent ${ }_{i}$ can acquire a piece of knowledge from Agent ${ }_{j}$, and then produce a new piece of knowledge (i.e., through knowledge modification or generation). Agent ${ }_{j}$ can learn that knowledge, and produce another piece of knowledge. Agent ${ }_{i}$ and Agent ${ }_{j}$ can also interact with other agents and their knowledge mutually evolves. One-many learning can result in common learning (Example 2-25). Common learning is the kind of learning in which all the agents in a team learn the same piece of knowledge (see Fig. 8). The knowledge sources for learning can be from agents or the environment (e.g., past design diagrams).

Figure 9 describes the distributions of the forms of collective learning in the three meeting sessions. It seems that most of the collective learning is in the form of one-one learning, averaging $63 \%$.

With respect to goal of learning, the two forms of learning, assisted and joint, were observed (see Examples 2-29 and 2-30). With respect to the involvement of an agent, not necessary to be involved and must be involved learning were identified (see Examples 2-31 and 2-32). Hence, the test results support Hypothesis 2.3: forms of collective learning exist.

It was observed that collective learning is interconnected with collective memory. What was learned collectively was used for current or future design practice or 


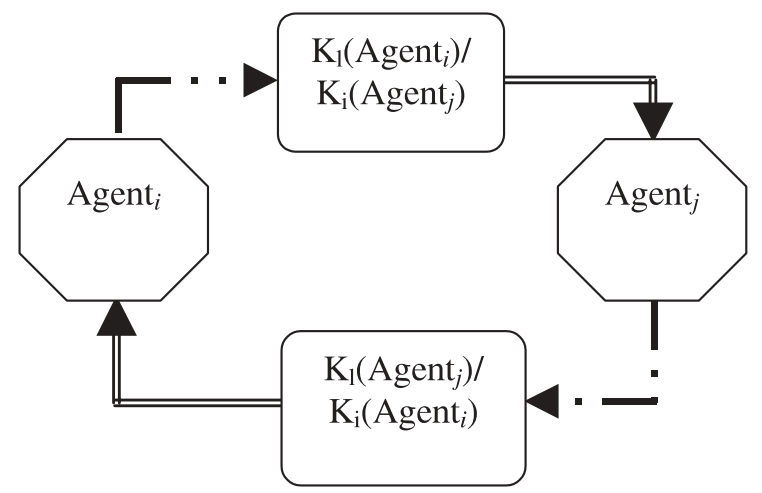

Key:
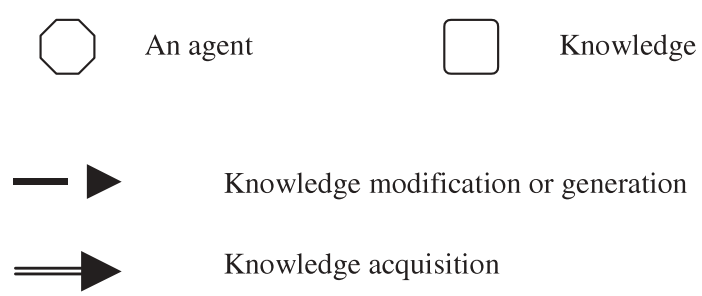

$\mathrm{K}_{\mathrm{l}}\left(\right.$ Agent $\left._{i}\right)$ - learned knowledge of agent ${ }_{i} ; \mathrm{K}_{\mathrm{l}}\left(\right.$ Agent $\left._{j}\right)$ - learned knowledge of agent $_{j} ; \mathrm{K}_{\mathrm{i}}\left(\right.$ Agent $\left._{j}\right)$ - input knowledge of agent ${ }_{j}, \mathrm{~K}_{\mathrm{i}}\left(\right.$ Agent $\left._{i}\right)$ - input knowledge of agent $; ; i, j=1, \ldots, n$ ( $n$ is the number of agents that perform mutual learning).

Fig. 7. Mutual learning.

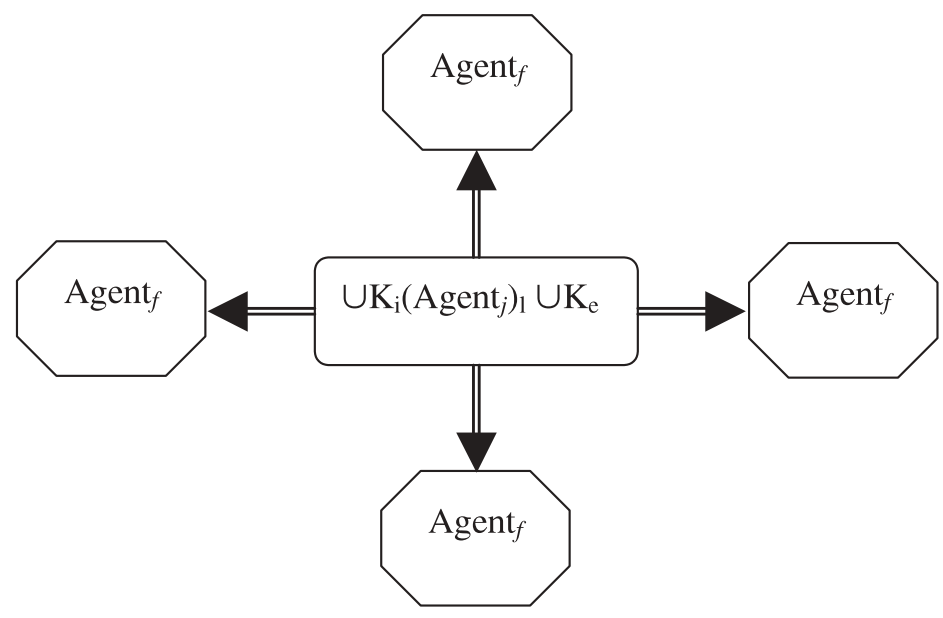

Key:

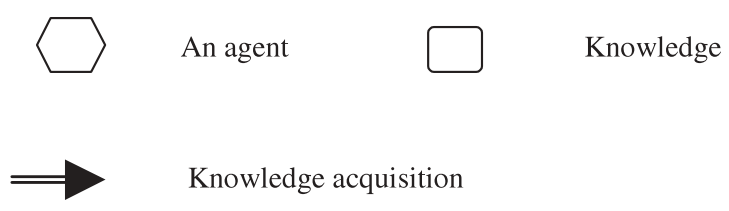

Agent $_{f}$ - Agent $f(f=1, \ldots, m ; m$ is the number of agents who perform a learning activities); $\cup \mathrm{K}_{\mathrm{i}}\left(\text { Agent }_{j}\right)_{1} \cup \mathrm{K}_{\mathrm{e}}$ - the union of input knowledge from agents and environment for learning $(j=1, \ldots, n ; n$ is the number of agents who provide input knowledge for learning).

Fig. 8. Common learning. 

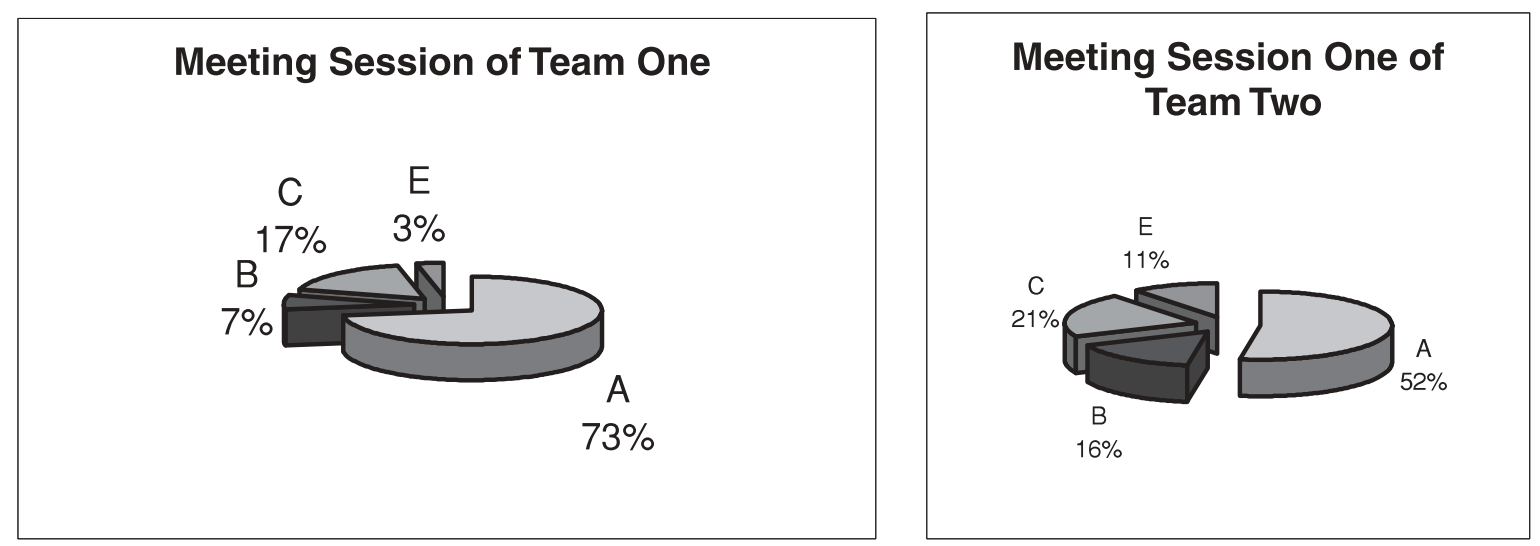

\section{Meeting Session Two of Team Two}

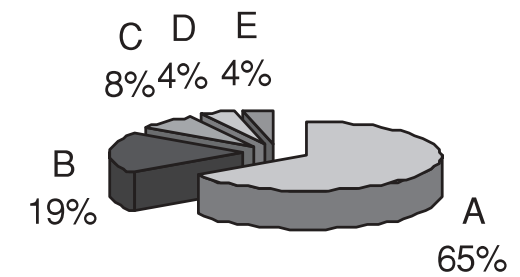

Key:

A: One-to-one learning

B: One-to-many learning

C: Many-to-one learning

D: Many-to-many learning

E: Derived forms

Fig. 9. The distributions of the forms of collective learning in the three teams.

learning activities, which indicates the existence of collective memory (see Examples 2-33, 2-34, and 2-35). Hence, the observed examples support Hypothesis 2.4 regarding the existence of collective memory and the interconnection between collective learning and collective memory. Memories composed of collective memory can be complementary, alternative, and conflicting, indicated from Table 4 and Table 5 with complementary and conflicting input knowledge for a design activity. In Table 4, GM added the complementary knowledge that a rest was needed for the plastic top to MH's knowledge of making a plastic top. In Table 5, the input knowledge from the client of the design team and those from the diagrams are conflicting, indicating from NB's verbalization "he was saying you can put them on the bottom" and "but see when he was looking at that one here (the diagram), think he might have seen that as a drop like that but it's not-it's all the way round." The client suggested that the heating elements can be dropped to the bottom of the tank directly from the top, while, indicated from the diagram, the heating elements should be put into the bottom through a hole in the bottom.

\subsection{Their links}

The three types of links (i.e., teleological, rationale, and epistemic) were observed in the meeting sessions.
The learning goal and the design goal interact with each other in two ways: the learning goal can precede the design goal, or vice versa. Table 6 illustrates an example in which GM's learning goal to learn the way to control the flow precedes the team's design goal of designing a way to con-

Table 4. An example of input knowledge from more than one designer

\begin{tabular}{l}
\hline No. Transcript \\
\hline $6 \quad$ GM: We still have to design this little lip bit to go on this. I think \\
it's the space that's the problem. How do we make something \\
that small that's going to be sealed properly and fit 2 tubes \\
in? Because we actually have to cut a bit of plastic. \\
DB: ... What about a lift top or plastic top. \\
GM: Just have it rubber to start with. \\
DB: That way we can make it seal. \\
MH: Just have a plastic top and make a thing like that out of \\
Glastic. Like dead thin and just stick the tubes through. \\
MH: That's what I mean. It's easy to put a lid on-it just needs a \\
square of plastic and glue it on.
\end{tabular}

The italic words represent the key words, phrases, and sentences used to identify the elements of a team design activity and a collective learning activity, which is applied to the rest of the protocols. 
Table 5. An example of conflicting input knowledge

\begin{tabular}{l}
\hline No. \\
\hline $8 \quad$ KS: Have you got the diagrams there? Because see the one that \\
NB: I wasn't entirely sure what he was talking about because he \\
was saying you can put them on the bottom and they were \\
2D like that. \\
CF: It was this one here ... these elements here should have \\
been down in that gap. \\
NB: But see when he was looking at that one here, I think he \\
might have seen that as a drop like that but it's not-it's all \\
the way round. \\
KS: He said that one would have less elements than that one. And \\
the heat elements aren't even in that one!
\end{tabular}

trol the flow. Table 7 depicts that the team's design goal to seal the entry point of the tube precedes PH's learning goal to learn the way to keep the tube tight.

There are reasons that trigger collective learning during the team design process (i.e., rationale triggers), which include explanation from other agents, confirmation from other agents, agreement between the agents, disagreement between the agents, failed design experience, and successful design experience. Each of the rationale triggers is illustrated with examples in Section A.2.2. As such, there is a rationale link between team design and collective learning.

Two types of learning operators have been identified, that is, knowledge acquisition with or without knowledge transformation. Three types of knowledge transformers observed, which include discovery, derivation, and specialization. Examples of knowledge transformation have been described in Section A.2.2. During a team design process, knowledge acquisition and transformation between agents occurred with different types of knowledge learned (see

Table 6. An example of input knowledge from two designers

\begin{tabular}{l} 
No. Transcript \\
\hline 7 \\
GM: Is there any way that we can incorporate the valve that \\
operates and that is also run by the processor in the \\
computer? So that when that is reaching out it allows the \\
flow to reach in. \\
GM: Is there any other way to do it when it is being controlled? \\
MH: Just put a valve on it or something. \\
PH: You can control the reservoir as it is; we talk about having \\
an injection system rather have a valve on it, having a \\
controller and the plunger to have a certain amount coming \\
out at one time. \\
GM: You are not controlling the level at the cartridge. \\
PH: pressurized. \\
PH: Yeahe at the top in the tank?
\end{tabular}

GM: Like at the top in the tank? 


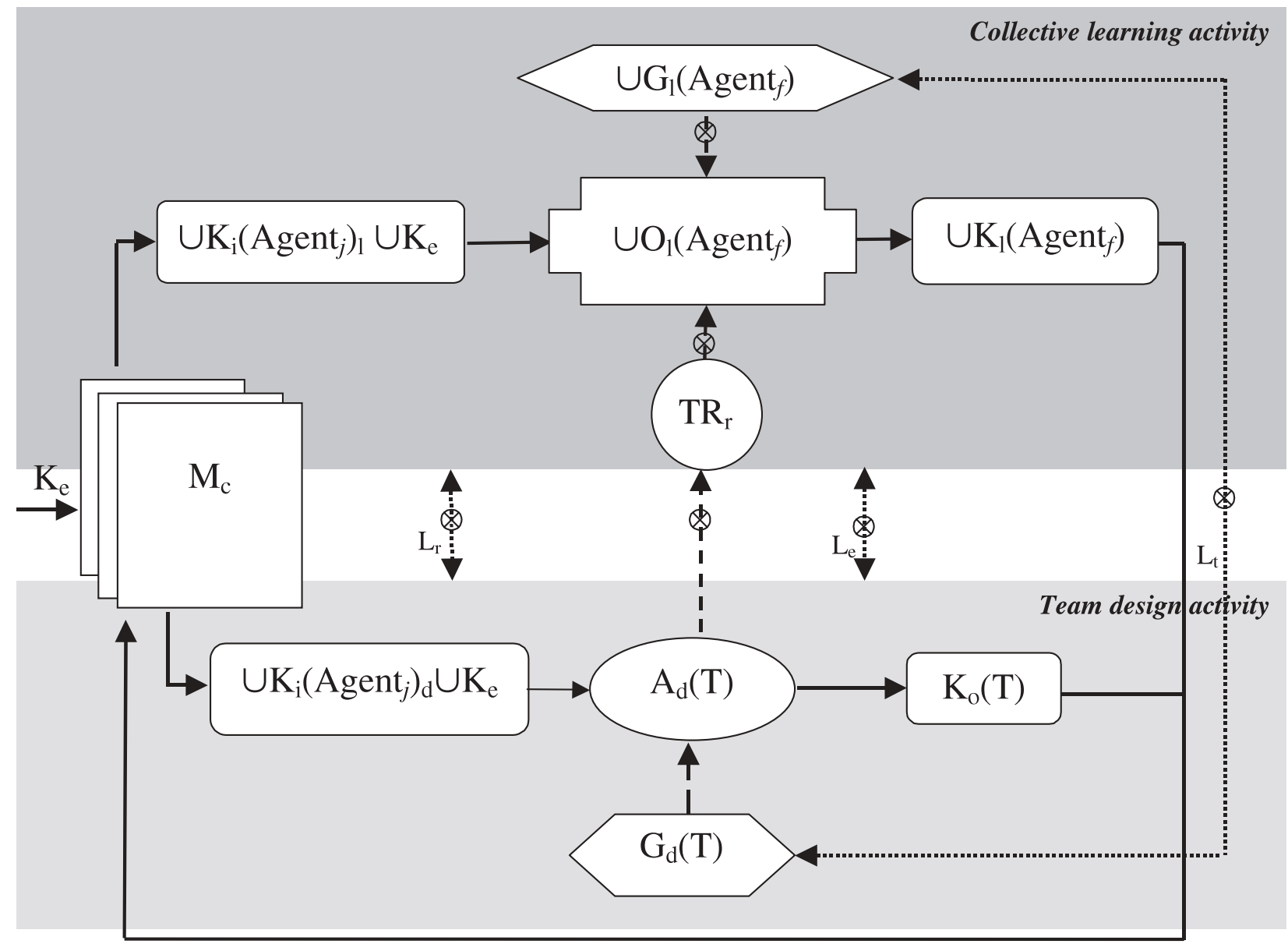

Key:

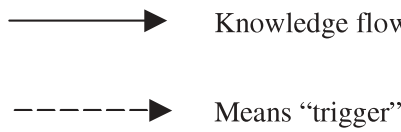

A memory

An activity links between team design and collective learning

$\otimes \quad$ Means "may not exist"

Knowledge

A goal

An event

\begin{abstract}
$\mathrm{M}_{\mathrm{c}}$ - collective memory; $\cup \mathrm{K}_{\mathrm{i}}\left(\text { Agent }_{j}\right)_{\mathrm{d}} \cup \mathrm{K}_{\mathrm{c}}$ - union of input knowledge from agents and external environment for team design activity; $\cup \mathrm{K}_{\mathrm{i}}\left(\mathrm{Agent}_{\mathrm{j}}\right)_{1} \cup \mathrm{K}_{\mathrm{e}}$ - union of input knowledge from agents and environment for collective learning; $\mathrm{O}_{1}\left(\right.$ Agent $\left._{f}\right)$ - learning operator of $\operatorname{agent}_{f}(f=1, \ldots, m$, where $m$ is the number of agents who perform learning activity); $\mathrm{G}_{1}\left(\right.$ Agent $\left._{f}\right)$ - the learning goal of agent $f_{f}(f=1, \ldots, m$, where $m$ is the number of agents who perform learning activity); $\mathrm{G}_{\mathrm{d}}(\mathrm{T})$ - team design goal; $\mathrm{A}_{\mathrm{d}}(\mathrm{T})$ - team design activity; $\mathrm{TR}_{\mathrm{r}}$ - rational trigger; $\mathrm{K}_{\mathrm{l}}\left(\mathrm{Agent}_{f}\right)$ - learned knowledge of $\operatorname{agent}_{f}\left(f=1, \ldots, m, m\right.$ is the agents who perform learning activity); $\mathrm{K}_{\mathrm{o}}(\mathrm{T})$ - output knowledge of team design; $\mathrm{L}_{\mathrm{r}}-$ Rational link; $\mathrm{L}_{\mathrm{e}}-$ Epistemic link; $\mathrm{L}_{\mathrm{t}}-$ Teleological link.
\end{abstract}

Fig. 10. A model of collective learning in design.

team design goal that represents the purpose of a design activity and directs it.

\subsubsection{Elements of a collective learning activity}

The model reveals that collective learning exists in team design, although not all team design activities were linked with a learning activity. Agents acquire and transform knowledge from each other through their interactions (e.g., team meetings). The elements of a collective learning activity are modeled as input knowledge, learning goal, rationale trigger, learning operator, and learned knowledge. 
One agent or more can learn from others and the environment through their interactions. Hence, input knowledge is the union of input knowledge of agents and the environment, which can include input knowledge from the agent who is performing the learning activity. Input knowledge for a learning activity can be part of or the same as those for the design activity, indicating the intrinsic links between design and learning.

A learning goal represents the learning purpose of one agent or a group of agents, which can trigger a learning activity and originates from a design process. However, not all learning activities have a learning goal.

Rationale triggers are the reasons that trigger a learning activity. The types of rationale triggers were identified as the following:

1. Explanation from other agents: Agents can learn from others with their explanations.

2. Confirmation from other agents: The confirmation of a piece of information or ideas can trigger others' to learn.

3. Agreement within agents: Agreed design ideas within some agents could result in others to learn.

4. Disagreement within agents: Agents can learn the potential failures of their design ideas because of the disagreement by other agents.

5. Successful design experience: Successful design experience in past designs can be learned and used for solving current problems, or current successful design experience can be used for future design practice.

6. Failure of design experience: Agents can learn lessons in their designs and thus avoid them in future design.

However, not all learning activities had a rationale trigger. Some agents learned from others only because other agents verbalized some knowledge and they acquired or transformed that knowledge.

Learning operator in a collective learning activity has the functionality of knowledge acquisition and transformation between different agents. Learning operators reside in those agents who perform a learning activity. When knowledge transformation occurs, the learning transformers described in Table 1 can be applied. In the experiments, the observed knowledge transformers included discovery, derivation, and specialization. It should be noted that there could be more than one agent who learns and might have different learning operators.

Types of knowledge can be learned, which have been observed in the experiments (see Fig. 6). Two types of knowledge were identified that can only be learned collectively, namely, common knowledge and knowledge of knowledge states of other agents. In a collective learning activity, two or more agents can learn, and thus the learned knowledge is union of learned knowledge from all the agents who learn.

\subsubsection{Relations between team design and collective learning}

Team design and collective learning can be described as cyclic in nature. Agents learn collectively during the design process. What is learned is stored in collective memory and can be used for future or current design practice.

A learning activity and a design activity cannot be separated in some situations. The input knowledge and learned knowledge of a learning activity can be part of or the same as the input knowledge and output knowledge of a design activity.

Three possible links between team design and collective learning exist: teleological, rationale, and epistemic.

\subsection{Forms of learning}

Agents in a team interact in different ways, which can result in different forms of learning. Forms of learning are classified according to agent interaction, goal of learning, and the involvement of an agent.

With respect to agent interaction, the basic forms of collective learning were identified as one-one, one-many, many-one, and many-many learning. Other forms can be derived from them. For example, one-one-many is the combination of one-one learning and one-many learning. Oneone learning can result in mutual learning, while one-many learning can result in common learning.

With the goal of learning considered, learning activities can be classified as assisted learning and joint learning. According to an agent's involvement, collective learning is classified as "not necessary to be involved" learning and "must be involved" learning.

\section{DISCUSSION}

The model presented in this paper describes the phenomenon of collective learning in team design and reveals the elements of team design and collective learning, the forms of collective learning, and their links. However, the weaknesses of the model were also realized. The types of learned knowledge, rationale triggers, and knowledge transformers identified within the model may not be exhaustive, and with other protocol analysis, other types can be identified. Their identification depends on both the design context and the relatively subjective judgement of the encoder of the protocol data.

Protocol analysis is considered as an effective way to investigate the cognitive behaviors of designers (Cross et al., 1996). In this paper, interviews were used as an assisted means to justify the encoded data, which to some degree have improved the protocol analysis approach. However, the weaknesses of protocol analysis have been identified, which include the following:

1. Although key words, phrases, and sentences are used to identify the elements of team design and collective 
learning, subjectivity of the analysis still exists. The identification of the rationale triggers and the knowledge transformers relied on the inference of the encoder, based upon the available information (e.g., input knowledge, output knowledge, and the design context).

2. Collective learning might exist during the design process; however, it might not have been identified. Some agents might learn from others; however, it was not reflected in the verbal data if they remained quiet.

3. The protocol analysis approach relied on the honesty of the participants. It was assumed that the participants verbalized their true belief during their discussions. If the participants did not verbalize their true beliefs, it would result in misleading analysis results (e.g., the false identification of collective learning activities).

In this research, student design teams were used. Collective learning in professional design teams might be different from those in student design teams. Students are still in their learning stage and generally have limited work experience. The way students work is different from the way that professional designers work. As such, the differences between the models of collective learning of student teams and professional design teams can be reflected in the elements of team design and collective learning activities, links between them, forms of collective learning, and manners of interactions. Whether the proposed model could be applied to professional design teams needs further investigation.

In this paper, the research of learning in design is extended from the design context of one agent to a design team. For a design project, design often involves multiple teams collaborating in the process to share expertise, resources, or responsibilities (Chiu, 2002). Collective learning can be further extended to the investigation of how teams as units learn from each other, and how collective learning within a team is linked with collective learning between teams. The insights on how learning occurs between design teams may have benefits in aspects, such as the improvement of team interactions, and the capture and utilization of knowledge between design teams.

Recently, research in creativity in design has drawn researchers' attention, such as that by Liu (2000), Saunders and Gero (2001), Gero (2002), and Tang and Gero (2002). The Workshop on Learning and Creativity in Artificial Intelligence in Design'02 (Duffy \& Wu, 2002) concluded that there is no consensus about the link between these two activities. The participants of the workshop were divided into two groups with different results produced. Group 1 concluded that creativity and learning are not connected. They are related to different dimensions of the design process. Creativity is a process related to unexpectedness, while learning is an acquisition process to acquire or extend the competence. Group 2 argued that creativity and learning are linked with each other. Creative design activity can cre- ate new knowledge, and a learning activity can contain creativity. Thus, in the future, the links between them can be further investigated, which can bring insights to both creativity and collective learning.

A number of agent-based learning systems have been developed in DAI, such as those in Haynes and Sen (1996), Sen and Sekaran (1996), and Prasad (1997). Relatively little research can be found in the domain of product design, such as the work of Grecu and Brown (1996a, 1996c). The requirements for an agent-based learning system in design and those in DAI can be different from those in DAI. The model developed in this paper can be used as a basis to develop agent-based learning systems in design.

\section{CONCLUSION}

In this paper, an investigation into the nature of collective learning in design is presented. A model of collective learning (see Fig. 10) is derived from the test results of the hypotheses, which explains the existence of collective memory, the elements of team design and collective learning, forms of learning, and the relations between team design and collective learning. Collective memory is the place where knowledge is stored and can be retrieved for team design and collective learning. The elements of a team design activity can be modeled as input knowledge, design goal, and learning knowledge, while a collective learning activity can consist of elements of input knowledge, learning operator, learned knowledge, learning goal, and rationale trigger. Forms of collective learning are classified according to agent interactions (e.g., one-one, one-many, many-one, manymany, and one-one-many learning), the goal of learning (e.g., assisted learning, and joint learning), and the involvement of an agent (e.g., must be involved learning and not necessary to be involved learning). Team design and collective learning can be described as cyclic in nature. In some situations, a learning activity and a design activity cannot be separated. There are three possible links between team design and collective learning, namely, teleological, epistemic, and rationale.

The model of collective learning can serve as a foundation for the development of computational means to support collaborative design and collective learning. The implications from the model to develop computer tools are identified as the following:

1. Computational agents may be equipped with the capability of collective learning. It was observed that collective learning exists between human designers. It is assumed that computational agent with learning capability will be developed and that collective learning will occur between computational agents or between human designers and computational agents.

2. The basic components for a design system to support collective learning will include mechanisms to enable knowledge input by different agents, knowledge acqui- 
sition and transformation between different agents, knowledge storage and retrieval within and between agents, and learning triggers (i.e., the learning goal and rational trigger) to start learning tasks.

3. Different forms of collective learning can be implemented. The implementation of forms of collective learning (e.g., mutual learning and common learning) can result in mutual knowledge evolution and the generation of common knowledge.

In the future, the research can be further extended to model collective learning between design teams, to investigate the links between collective learning and creativity, and to develop agent-based learning systems in design with the proposed model as a basis.

\section{ACKNOWLEDGMENTS}

The authors express their appreciation to all the team members involved in the experiments and who helped check the encoded data. We are also indebted to Mrs. C. Rankin and Ms. A. Stevenson, who spent many hours transcribing the tapes. We are thankful to the University of Strathclyde, which provided a 3-year studentship to support this work. Finally, the authors are indebted to the anonymous reviewers' insightful comments and feedback on this paper and their time and effort spent on it.

\section{REFERENCES}

Argyris, C., \& Schön, D. (1978). Organisational Learning: A Theory of Action Perspective. Reading, MA: Addison-Wesley.

Ashcraft, M. (1994). Cognition. Englewood Cliffs, NJ: Prentice-Hall.

Chiu, M. (2002). An organisational view of design communication in design collaboration. Design Studies 23, 197-210.

Cross, N., Christiaans, H., \& Dorst, K. (1996). Introduction: the Delft protocols workshop. In Analysing Design Activity, pp. 1-14. New York: Wiley.

Cross, N., \& Nathenson, M. (1981). Design methods and learning methods. In Design: Science: Method (Jacques, R., \& Powell, J., Eds.), pp. 281-296. Guildford, UK: IPC Science and Technology Press.

Cross, R., \& Israelit, S. (2000). Introduction: strategic learning in a knowledge economy. In Strategic Learning in a Knowledge Economy: Individual, Collective and Organizational Learning Process (Cross, R., \& Israelit, S., Eds.). London: Butterworth Heinemann.

Dewey, J. (1938). Experience and Education. London: Collier-Macmillan.

Dillenbourg, P. (1999). Introduction: What do you mean by "collaborative learning"? In Collaborative Learning: Cognitive and Computational Approaches (Dillenbourg, P., Ed.), pp. 1-19. New York: Pergamon.

Duffy, A., \& Wu, Z., Eds. (2002). AID'02 Workshop: Learning and Creativity. Cambridge: CAD Centre, University of Strathclyde.

Duffy, S., \& Duffy, A. (1996). Sharing the learning activity using intelligent CAD. Artificial Intelligence for Engineering Design, Analysis and Manufacturing 10, 83-100.

Eastman, C. (1970). On the analysis of intuitive design processes. In Emerging Methods in Environmental Design and Planning (Moor, G., Ed.). Cambridge, MA: MIT Press.

Fricke, G. (1996). Successful individual approaches in engineering design. Research in Engineering Design 8, 151-165.

Gero, J. (1990). Design prototypes: a knowledge representation schema for design. AI Magazine 11(4), 26-36.

Gero, J. (2002). Computational models of creative designing based on situated cognition. In Creativity and Cognition (Hewett, T., \& Kavanagh, T., Eds.), pp. 3-10. New York: ACM Press.

Gero, J., \& Kannengiesser, U. (2002). The situated function-behaviourstructure framework. In $A I D^{\prime} 02$, pp. 89-104. Cambridge: Kluwer Academic.
Gero, J., \& Neill, T. (1998). An approach to the analysis of design protocols. Design Studies 19(1), 21-61.

Grecu, D., \& Brown, D.C. (1996a). Design agents that learn. Artificial Intelligence for Engineering Design, Analysis and Manufacturing 10, 149-150.

Grecu, D., \& Brown, D.C. (1996b). Dimensions of learning in agent-based design. Artificial Intelligence in Design' 96 ML in Design Workshop.

Grecu, D., \& Brown, D.C. (1996c). Learning by single function agents during spring design. Artificial Intelligence in Design '96, pp. 409 428. Boston: Kluwer Academic.

Grecu, D., \& Brown, D.C. (1998). Dimensions of learning in design. Artificial Intelligence for Engineering Design, Analysis and Manufacturing 12, 117-122.

Hare, A. (1976). Handbook of Small Group Research. New York: Free Press.

Haynes, T., \& Sen, S. (1996). Co-adaptation in A Team. International Journal of Computational Intelligence and Organizations 1(4), 46-62.

Hubka, V., \& Eder, W. (1996). Design Science. New York: Springer-Verlag.

Kelly, G. (1955). The Psychology of Personal Constructs. New York: Norton.

Kim, D. (1993). The link between individual and organizational learning. Sloan Management Review Fall, 37-50.

Kocabas, S. (1991). A review of learning. The Knowledge Engineering Review 6(3), 195-222.

Kofman, F. (1992). Lecture Slides. Cambridge, MA: MIT Sloan School of Management. Quoted in D. Kim's paper. Sloan Management Review, Fall, p. 39.

Kolb, D. (1984). Experiential Learning. London: Prentice-Hall.

Levin, P. (1966). The design process in planning. Town Planning Review $37(1), 5-20$.

Lewin, K. (1951). Field Theory in Social Sciences. New York: Harper \& Row.

Liu, Y. (2000). Creativity or novelty? Design Studies 21, 261-276.

Marples, D. (1960). The Decisions of Engineering Design. London: Institute of Engineering Designers.

Marton, F., \& Booth, S. (1997). Learning and Awareness. Hillsdale, NJ: Erlbaum.

Mason, E. (1970). Collaborative Learning. London: Ward Lock Educational.

Neergaard, C. (1994). Creating a learning organisation: a comprehensive framework. PhD Thesis. Denmark: Department of Production, Aalborg University.

Persidis, A., \& Duffy, A. (1991). Learning in engineering design. In Intelligent CAD, III (Yoshikawa, H., Tomiyama, T., \& Arbab, F., Eds.) Amsterdam: Elsevier Science Publishers B.V.

Piaget, J. (1970). Genetic Epistemology. New York: Columbia University Press.

Prasad, M. (1997). Learning organizational roles in a heterogeneous multiagent system. International Journal of Human-Computer Studies 48(1), 51-67.

Radcliffe, D. (1996). Concurrency of actions, ideas and knowledge displays within a design team. In Analysing Design Activity (Cross, N., Christiaans, H., \& Dorst, K., Eds.), pp. 343-364. West Sussex: Wiley.

Reich, Y. (1998). Learning in design: from characterizing dimensions to working systems. Artificial Intelligence for Engineering Design, Analysis and Manufacturing 12, 161-172.

Saunders, R., \& Gero, J. (2001). Artificial creativity: a synthetic approach to the study of creative behaviour. In Computational and Cognitive Models of Creative Design (Gero, J., \& Maher, M., Eds.), Vol. V, pp. 113-139. Sydney, Australia: University of Australia, Key Centre of Design Computing and Cognition.

Schank, R. (1982). Dynamic Memory: A Theory of Reminding and Learning in Computers and People. Cambridge: Cambridge University Press.

Sen, S., \& Sekaran, M. (1996). Multiagent coordination with learning classifier systems. In Adaption and Learning in Multi-agent Systems, pp. 218-233. New York: Springer-Verlag.

Sim, S. (2000). Modelling learning in design. PhD Thesis. Glasgow: University of Strathclyde, CAD Centre, Department of Design, Manufacturing and Engineering Management.

Stumpf, S., \& McDonnell, J. (2001). Individual learning styles and perceptions of experiential learning in design teams. Proc. Design Thinking Research Symp. 5 (DTRS2001) Designing in Context. Delft: Delft University of Technology.

Stumpf, S., \& McDonnell, J. (2002). Talking about team framing: using argumentation to analyse and support experiential learning in early design episodes. Design Studies 23(1), 5-23. 
Tang, H., \& Gero, J. (2002). A cognitive method to measure potential creativity in designing. Workshop 17-Creative Systems: Approaches to Creativity in AI and Cognitive Science, ECAI-02, pp. 47-54, Lyon.

West, M. (1994). Effective Teamwork. Leicester: The British Psychological Society.

Zhang, Y. (1998). Computer-based modelling and management for current working knowledge evolution support. $\mathrm{PhD}$ Thesis. Glasgow: University of Strathclyde, Department of Design, Manufacturing and Engineering Management.

Zhichao Wu is a $\mathrm{PhD}$ candidate at the Computer Aided Design Centre, Department of Design Manufacture and Engineering Management, University of Strathclyde. He holds a BS degree in civil engineering and an MS degree in project management. His research interests include learning in design, situatedness, agent-based design, distributed design, and system integration. He coorganized the Workshop on Learning and Creativity at the Seventh International Conference on Artificial Intelligence in Design in 2002.

Alex H.B. Duffy completed an apprenticeship as a shipwright draughtsman and a further 2 years in shipbuilding before he obtained a BS in naval architecture and a $\mathrm{PhD}$ in knowledge-based computer support for conceptual engineering design. He is presently the Director of the Computer Aided Design Centre and the Director of Research in the Department of Design Manufacture and Engineering Management at the University of Strathclyde. Dr. Duffy's main research interests have been the application of knowledge-based techniques in early-stage design, product and domain knowledge modeling, learning in design and reuse, performance measurement and design productivity, process optimization, vague geometric modeling and sketching support, and design coordination.

\section{APPENDIX A: EXAMPLES OF OBSERVATIONS}

\section{A.1. Team design}

\section{A.1.1. Design goal}

Team design goals in many design activities seem to be verbalized at the beginning of the protocol segment. The key words used to identify team design goals were like "We need to design a lid ..." (GM), "We need to design something to seal the tube ..." (GM), and "I still think we need something to stop the flow ..." $(\mathrm{MH})$, in which the design goals were inferred as designing a lid, designing something to seal the tube, and designing something to stop the flow. (GM represents the agent who verbalized the words.) Team design goals can also be inferred from the questions verbalized by the designers. For example, from the questions "Right, what about the mounting of the tank then? Do you think it should be on the gantry or moving with it?" (GM), the team design goal is identified as deciding the location to mount the tank. Different design goals are identified in the teams, such as designing the insulation for the tank, designing the location of the inner tank, and designing the size of the tank.
Table A.1. An example of input knowledge from one designer only

\begin{tabular}{ll}
\hline \hline No. & \multicolumn{1}{c}{ Transcript } \\
\hline 3 & MH: \\
& WMas it sealed though? \\
& No ..., which was part of it but even then it shouldn't, \\
& because it was totally pouring out like. \\
& $\begin{array}{l}\text { But even it was sealed. It probably put too much pressure on } \\
\text { the thing. Because it was totally flooding out at the rate at } \\
\text { which it just comes out normally. }\end{array}$
\end{tabular}

\section{A.1.2. Input knowledge}

EXAMPLE 1-1. Only one agent provides input knowledge for a team design activity.

In the example in Table A.1, only GM provides input knowledge that the cartridge will not be sealed because the fluid will pour out.

EXAMPle 1-2. More than one agent provides input knowledge for a team design activity.

Table 4 illustrates one example with input knowledge from three designers (i.e., GM, DB, and MH) in the design activity of exploring solutions in designing the lid of the cartridge.

Example 1-3. The input knowledge from two agents or more is complementary.

In the example in Table 4, GM added the complementary knowledge that a rest was needed for the plastic top to MH's knowledge of making a plastic top.

ExAmple 1-4. The input knowledge is alternative.

Table 4 shows alternative solutions from GM, DB, and MH in designing the lid of the cartridge. GM had the input knowledge of having a rubber top, implied from her verbalization "just have it rubber to start with." DB had the input knowledge of having a lift top or plastic top, indicated from "... What about a lift top or plastic top." MH had the idea of making a plastic top with a rest, indicated from "just have a plastic top and make a thing like that out of plastic ..." and "that's what I mean. It's easy to put a lid on-it just needs a square of plastic and glue it on."

EXAMPLE 1-5. The input knowledge is conflicting.

In Table 5, the input knowledge from the client of the design team and those from the diagrams are conflicting, indicating from NB's verbalization "he was saying you can put them on the bottom" and "but see when he was looking at that one here (the diagram), think he might have seen that as a drop like that but it's not-it's all the way round." The client suggested that the heating elements can be dropped to the bottom of the tank directly from the top, while, indicated from the diagram, the heating elements should be put into the bottom through a hole in the bottom.

\section{A.1.3. Output knowledge}

The identification of output knowledge could be inferred or derived from the input knowledge of agents and their design goals. 
Table A.2. Collective learning inferred from the context of the verbal data

\begin{aligned} & \hline \hline No. \multicolumn{1}{c}{ Transcript } \\ & \hline $18 \quad$ GM: That doesn't have to be sealed (pointing to drawing) because \\ & as long as that water level's there, you're not gonna' get any \\ & water in it. They're not gonna' get any air in it rather. As \\ & long as it's down to like there, know what I mean, as long as \\ & that bit's covered. \\ & DB: Even there, if it's running through a sponge then you're not \\ & gonna' get any air through it anyway. \\ & PH: Don't want it contaminated with dust as well, you know you \\ & want to keep it quite ... \\ & GM: Aye, it would definitely have to be a sealed... \end{aligned}

The output knowledge in the design activity in Table A.1 (i.e., the cartridge should not be sealed) was derived from the input knowledge from GM (i.e., the fluid will pour out and the cartridge should not be sealed), indicated from "no ..., which was part of it but even then it shouldn't, because it was totally pouring out like," and the design goal (i.e., deciding whether the cartridge should be sealed or not), indicated from MH's verbalization "Was it sealed though?." Table 4 illustrates an example that the output knowledge (i.e., ways to design the lid of the cartridge) is inferred from the input knowledge of the three designers (i.e., GM, DB, and $\mathrm{MH}$; see Section A.1.2) and their design goal (i.e., designing the lid of the cartridge).

\section{A.2. Collective learning}

\section{A.2.1. Identification}

Some key words, phrases, or sentences can explicitly indicate that collective learning occurs, such as the following:

1. "Because Gerry said so." (GM);

2. "Aye, I get that bit." (GM) "Do you understand that Gillian?" (PH) "Yeah." (GM)

3. "Do you know what I mean?" (MH) "Yeah." (GM)

4. "Right, see now I understand what the diagram (i.e., a diagram drawn by DB) is where I didn't actually really know." (GM)

The protocol in Example 1 indicates that GM learned a piece of knowledge from Gerry. Example 2 implies that GM learned a design idea from PH. Example 3 indicates that GM learned a piece of knowledge from MH. From Example 4, it can be inferred that GM learned from DB. In some examples, collective learning was identified by some key words, phrases, or sentences that implicitly indicate the occurrence of collective learning. The sentence, "Paul sketched one the other day and it is just like a rack and pinion type thing" (GM), indicates that GM learned the design idea from Paul. Collective learning can also be identified from the context of the verbal data. Table A. 2 shows that GM suggested that the cartridge did not have to be sealed with the key words "that doesn't have to be sealed." However, with PH's input knowledge that it would have the problem of contamination, identified by the key words "don't want it to be contaminated with dust as well," GM learned that the cartridge had to be sealed, indicated by her verbalization "aye, it would definitely have to be sealed ..." GM's knowledge state changed from the cartridge "doesn't have to be sealed" to "it would definitely have to be sealed."

\section{A.2.2. The elements}

Input knowledge.

EXAMPLE 2-1. Input knowledge for collective learning is provided by one agent.

MH's learning activity indicated in Table A.1 had the input knowledge from GM only. MH had the goal to learn whether the cartridge needed to be sealed with his verbalization "was it sealed though?" GM suggested that the cartridge should not be sealed, indicated from her verbalization "it shouldn't, because it was totally pouring out like." MH learned that the cartridge should not be sealed, inferred from his key words "but even if it was sealed. It is probably put too much pressure on the thing."

EXAMPLE 2-2. Input knowledge for collective learning is provided by two agents or more.

GM's learning activity inferred in Table 6 had input knowledge from two designers, $\mathrm{MH}$ and $\mathrm{PH}$. GM had the goal to learn the way to control the flow, inferred from her question "is there any way that we can incorporate the valve ..." and "is there any way to do it when it is being controlled." MH suggested using a valve to control the flow with his verbalization "just put valve on it or something," while PH had the idea to use an injection system with his key words "we talk about having an injection system rather than have a valve on it."

EXAMPLE 2-3. Input knowledge for collective learning is provided by other agents and the agents themselves.

In the example in Table A.3, GM's learned knowledge was derived from her own input knowledge and from PH's input knowledge. GM had the idea to design a porous cap, indicated from her key words "I think you'd better just having a porous cap." Then, PH suggested that they could have a filter as well with his key words "you can get filters as well you know." Based upon PH's input, GM derived the new idea that they could design a porous

Table A.3. Collective learning with input knowledge of other agents and the agent themselves

\begin{tabular}{ll}
\hline \hline No. & \multicolumn{1}{c}{ Transcript } \\
\hline 24 & GM: I think you'd be better just having a porous cap ... \\
& MH: Just make sure that that's always full and that solves the \\
problem. \\
PH: You can get filters as well you know. \\
MH: Just so you can fill it up at the top-like as long as it's so \\
full. \\
GM: I think that's better. I think having a cap with like a hole in \\
it which is some kind of filter or ... \\
PH: You get screws like that.
\end{tabular}


Table A.4. An example of input knowledge from other knowledge sources (e.g., drawings of past designs)

\begin{tabular}{l}
\hline No. Transcript \\
\hline 7 \\
KS: (pointing to the drawing) This will run in but curve round \\
the bottom of the tank? Because that's the other thing, \\
remember they mentioned you could just put the heating \\
elements in a circle and drop it over the dome base and it \\
would just find its own balance on it? \\
KS: Was that when he was talking about a ring . . \\
out. So you must then put them in some kind of casing as \\
well first. So you can't really just drop it over. \\
the gap at the bottom? There's going to be a gap at the \\
bottom where the heat elements can go and that was to put \\
the ring down there to hold. . \\
CF: The heating elements were just a shield pipe and he also \\
said that there would be 3-foot radius or diameter, I think \\
he meant radius, which is about a meter. So they would go \\
round a 2-meter diameter. What's the diameter of the bottom \\
of the tank? 3 meters.
\end{tabular}

cap with a filter in it, indicated from her sentence "I think having a cap with like a hole in it which is some kind of filter or ..."

EXAMPLE 2-4. Input knowledge in some learning activities can come from other sources (i.e., the diagrams of past designs) besides those from agents.

In the learning activity in Table A.4, KS, MM and CF assisted one another to learn the layout of the heating elements in the bottom of the tank from the diagram. In this example, KS, MM, and $\mathrm{CF}$ learned from the diagram with the interpretation of other agents and their own understandings. The diagram explains how the pipes go from the top of the tank to the bottom, and the layout of the heating elements in the bottom. Other agents had the input knowledge on the shape of the heating element and the size of the pipe.

EXAMPLE 2-5. The input knowledge for the collective learning activity can be the same as that for the design activity, which indicates that in some situations a learning activity and a design activity cannot be actually separated.

For example, in the protocol in Table 7, the input knowledge for the team design activity was the same as that for PH's learning activity. The goal of the design activity was to design a way to seal the tube, inferred from GM's verbalization "we need to design something to seal the tube so it doesn't leak." DB provided a way to seal the tube, using grub screws with a plastic sleeve around it, indicating from his verbalization "grub screws?" and "with a plastic sleeve around it." PH had the goal to learn how a grub screw can be used to seal the tube, inferred from his question "where do we put a grub screw at the side to keep it tight?" DB's input knowledge of using a plastic sleeve around the grub screw trigger him to learn. Through the design activity, PH learned that a grub screw with a plastic sleeve could be used to seal the tube.

ExAMPLE 2-6. The input knowledge for the collective learning activity can be part of that for the design activity.
Table A.5. Input knowledge of a learning activity being part of that of a design activity

\begin{tabular}{l}
\hline No. \\
\hline $12 \quad$ TManscript \\
PH: I think it should be sealed along those edges so that the \\
GM: What do you mean? \\
PH: Just a small gasket that seals around, but I don't know how \\
you'd get that done and how you'd seal it. \\
GM: Even just use silicon gel 'round the top. \\
DB: But because it's 3 compartments ... Easier if it's just a \\
single compartment. But because it's the three you'd have to \\
have rigid edges round here and a seal across ... \\
PH: Seal across here and here ... you could possibly have it \\
coming in ... \\
GM: Could you not have the silicon binder stuff just going along \\
all these surfaces and just stick the top on? \\
MH: If you cut it well enough you should get it pretty close. \\
MH: long as it's flat.
\end{tabular}

In the protocol in Table A.5, four designers GM, PH, MH, and DB provided input knowledge for the design activity to seal the cartridge. PH had the input knowledge of using a small gasket, indicated from "just a small gasket that seals around." GM suggested using silicon gel round the top, implied from her key words "even just use silicon gel around the top." DB suggested that the three compartments should be sealed across, indicated from his verbalization "because it's three you'd have to have rigid edges round here and a seal across ...," MH suggested that the edge should be cut flat so that the cartridge could be sealed, indicated from her verbalization "if you cut it well enough you should get it pretty close." However, the input knowledge for GM's learning activity only came from MH. GM had the goal to learn whether they can use silicon binder to seal the cartridge, indicated from her question "could you not have the silicon binder stuff just going along all these surfaces and just stick the top on?" MH suggested that if the surface was flat enough, it could be sealed by a silicon binder, indicating from his key sentences "if you cut it well enough you should get it pretty close" and "as long as it's flat." In this design activity, GM learned that if the surface of the cartridge was cut flat, they could use the silicon gel to seal the cartridge.

ExAmple 2-7. Some learning activities are not linked with a design activity in which there is only input knowledge for a learning activity identified.

For example, Table A.4 illustrates that KS, MM, and CF assisted one another to learn the layout of the heating elements in the tank in which there is no design activity identified. The key words "(pointing to the drawing) this will run in but curve round the bottom of the tank?" indicates that KS learned from the diagram the layout of the pipe but was not quite sure about it. The key words "remember they mentioned you could just put the heating elements in a circle and drop it over the dome-base and it would just find it's own balance on it?" implies that he also learned from their client the layout of the heating elements, however, he was still not sure about it. MM was not clear about the layout of the heating elements, indicated by the key words "was that when he 
Table A.6. An example of common knowledge

\begin{tabular}{l}
\hline No. \\
\hline $1 \quad$ GM: \\
$\begin{aligned} \text { Right, we have to use cartridge because we are using the } \\
\text { cradle. Because Kevin said so. So we have to design a little } \\
\text { bit to go on the top of this about this topic. } \\
\text { DB: }\end{aligned}$ \\
$\begin{array}{l}\text { Yeah. It is not just the tube into the ink recess or whatever it } \\
\text { MH: Yeah. }\end{array}$
\end{tabular}

was talking about a ring?" CF learned the layout of the heating elements from the client, inferred from "the heating elements were just a shield pipe and he also said that there would be three foot radius or diameter," which cleared KS and MM's vague idea. KS and MM learned from CF, because in the later design activity, they worked on the layout of the heating elements as a shield pipe. This learning activity was not directly linked with a design activity. Thus, there was no input knowledge for a design activity identified.

Learned knowledge.

Example 2-8. Common knowledge is learned.

The learned knowledge in Table A.6 that they used a cartridge because they were using the cradle, and that they needed to design the top of the cartridge was an example of common knowledge. In the subsequent design activities, they started to design the top of the cartridge. An agent can also learn what knowledge others have.

Example 2-9. One agent learns the knowledge states of others.

Table A.7 illustrates an example in which rather than learning the volume of fluid, MH learned that GM might know the volume.

EXAMPLE 2-10. Learned knowledge can be part of the output knowledge of a team design activity.

Table A.5 illustrates an example in which learned knowledge was part of the output knowledge of the design activity. GM learned that silicon binder could be used to seal the edges of the cartridge if they were cut flat, indicated from GM's verbalization "Could you not have the silicon binder stuff just going along all these surfaces and just stick the top on?" and MH's verbalization "if you cut it well enough you should get it pretty close" and "as long as it's flat," while the output knowledge of the team design activity was different ways to seal the edges of the cartridge.

Table A.7. An example of metaknowledge

\begin{tabular}{ll}
\hline No. & \multicolumn{1}{c}{ Transcript } \\
\hline 9 & GM: We need to do it as an experiment. \\
& MH/PH: I thought he did that. \\
GM: All he did is to give us the volume. \\
MH: What was the volume then? \\
GM: I've probably got it on an email somewhere. \\
MH: Because we can work it out from that.
\end{tabular}

EXAMPLE 2-11. Learned knowledge is the same as output knowledge of a team design activity.

Table A.1 illustrates an example in which learned knowledge was the same as the output knowledge of a design activity. $\mathrm{MH}$ learned that the cartridge should not be sealed, inferred from MH's verbalization "Was it sealed though?" and GM's verbalization "No ... which was part of it but even then it shouldn't, because it was totally pouring out like" and MH's second verbalization "But even it was sealed. It probably put too much pressure on the thing. Because it was totally flooding out at the rate at which it just comes out normally." The output knowledge of the design activity was the same as MH's learned knowledge.

Learning goal. It is difficult to identify learning goals, as the individuals did not verbalize such words as "the goal I learn from him/her is . .." However, learning goals can be identified through key words or phrases or sentences, and it could also be inferred from design goals, input knowledge, and learned knowledge. In the learning activity in Table A.1, the learning goal of $\mathrm{MH}$ on deciding whether the cartridge was sealed or not was identified based upon the sentence "Was it sealed though?" MH's learning goal in the protocol in Table A.8, learning the way to stop the flow, can be inferred from the key sentence "I still think we need to stop the flow," and the input knowledge of DB that a valve could be used and the input knowledge of GM that a pump was not needed. There are some learning activities without a learning goal. In these learning activities, designers learned from others only because others verbalized some knowledge. That is, learning occurred without an explicit goal. For example, within the protocol in Table A.9, it was identified that GM learned from PH the design idea of using a clip to attach the gadget to the cartridge from PH's key words "if you clip your print cartridge in and this is attached (holding gadget)" and GM's key word "yeah." There was no learning goal in this learning activity. GM learned from PH because PH verbalized his design idea during their discussions.

Learning goals can reflect the learning purpose of one agent or a group of agents. The verbalization "What bothered me at the presentation as well was the fact that it's not going to be standard pieces really, is it for the GRP [glass reinforced plastic material]?" (KS) implies KS's learning goal to know whether GRP was standardized in the components of the tank. The sentence "What's SI?" (CF) reflects CF's learning goal to know the meaning of the unit SI. The verbalization "I don't really think we know enough about the ... especially the manufacturing processes ..." (KS) indicates the learning goal of the whole team to know the manu-

Table A.8. An example of the identification of a learning goal

\begin{tabular}{l}
\hline \hline No. \\
\hline $6 \quad$ MH: I still think we need something to stop the flow. It will improve \\
it putting the sponge but they will still just get saturated. \\
DB: We could have a valve. \\
MH: We cannot just have a large bottle of water pouring into that \\
and just hoping it will cope. \\
DB: We don't think we need a pump. \\
DB: If it seals properly it will prevent the water.
\end{tabular}


Table A.9. Collective learning without learning goal identified

\begin{tabular}{ll}
\hline No. & \multicolumn{1}{c}{ Transcript } \\
\hline $15 \quad$ PH: & So what if you have it in two bits, what if you have a clip? If \\
& you clip your print cartridge in and this is attached (holding \\
& gadget). You know you have your bottle or whatever. It \\
& doesn't matter where your bottle is if its gravity feed you \\
& know you just need to sit it above. \\
GM: Yeah.
\end{tabular}

facturing processes of the tank. The verbalization "We need to know how much wool we're going to need, what diameter the tank's going to be?" (NB) implies the learning goal of the team to know the quantity of insulation wool and the size of the tank.

\section{Rationale trigger.}

EXAMPLE 2-12. One agent can learn from another through the agent's explanation of the rationale behind design decisions.

In Table A.10, GM learned PH's design idea by his explanation, indicated from GM's verbalization "(pointing) what is that?" and PH's key sentences "that's a hole" and "that is the hole for the tube so just tuck it in—snap fit, just push out."

EXAMPLE 2-13. One agent can learn, triggered by another's confirmation.

Table A.11 shows that AF's learning activity was triggered by KS's confirmation that the GRP was laid layer by layer on a wooden mold. AF was not quite sure the way to make GRP molds, indicating from "there might actually be pressings, I don't know. How do they make this GRP that you're kind of . . . is it actually being laid up?" KS confirmed with his verbalization "yeah, it is normally a wooden mould and then they put the different layers over it." AF learned the idea by reinterpreting it with his verbalization "they layer over it and then put the proxy on it and then ..."

EXAMPLE 2-14. The agreements between agents can trigger their learning.

In Table A.1, the agreement between $\mathrm{MH}$ and GM triggered MH's learning activity. MH agreed with GM that a cartridge should not be sealed because "it probably put too much pressure to the thing (flow)."

Table A.10. Other agents' explanations identified as a rationale trigger

\begin{tabular}{ll}
\hline \hline No. & \multicolumn{1}{c}{ Transcript } \\
\hline 21 & PH: \\
& (drawing) Set it onto two clips and just press it in. There you \\
& go; there's the design. \\
GM: (pointing) What's that? & PH: That's a hole. \\
PH: & $\begin{array}{l}\text { That's the hole for the tube so just tuck it in—-snap fit, just } \\
\text { push out. }\end{array}$ \\
GM: & Aye, I get that bit.
\end{tabular}

Table A.11. Confirmation from other agents identified as a rationale trigger

\begin{tabular}{l}
\hline \hline No. \\
\hline $10 \quad$ AF: There might actually be pressings; I don't know. How do \\
they make this GRP that you're kind of . . is it actually \\
being laid up? \\
KS: Yeah, it's normally a wooden mold and then they put the \\
different layers over it. \\
AF: The layers over it and then put the epoxy on it and then ...
\end{tabular}

ExAmPLE 2-15. The disagreements between agents can trigger their learning.

In Table A.12, the disagreement of GM triggered MH to learn that there was potential danger to make the printer run dry. $\mathrm{MH}$ suggested that they could use a sponge to control the flow with his words "or we need a way of soaking the sponge and then wait until it runs out." However, GM disagreed with it and realized that there might be the danger to let the printer run dry, indicated from her verbalization "the danger is that we let it run dry."

Example 2-16. Failed design experience can trigger learning.

Table A.2 illustrates that the failure of GM's design idea triggered herself to learn. PH explained that if the cartridge was not sealed it would have the problem of contamination.

Example 2-17. Successful design experience can trigger learning.

Table A.13 depicts that the successful design idea as the result of the knowledge evolution between GM and DB triggered $\mathrm{MH}$ to learn. DB had a vague idea to design the top of the cartridge, indicated from his key words "I'm trying to think of an example. You know the kind of thing I'm talking about." The idea became clear with GM's verbalization "yeah, like the steradent tops?" and DB's verbalization "aye, that idea." MH learned this successful idea, inferred from his verbalization "aye, that's a good idea."

Table A.12. Disagreement between agents identified as a rationale trigger

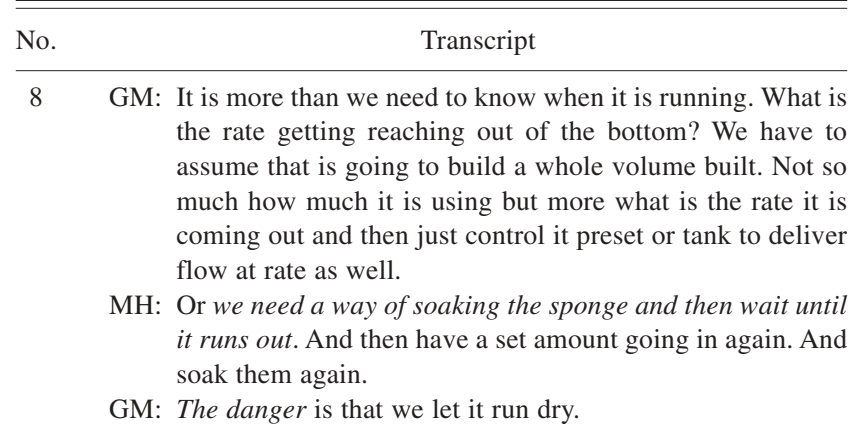


Table A.13. Successful design experience identified as a rationale trigger

\begin{tabular}{l} 
No. \\
\hline $26 \quad$ Transcript \\
GM: Yhat just fits in the top-I'm trying to think of an \\
DB: Aye, is that the stuff they use for dentures? \\
GM: Yeah. \\
DB: Aye, that idea. \\
MH: (drawing) Aye, like this-is it like that, is that what \\
you're talking about? And you push them back down. \\
DB/GM: Yeah. \\
MH: Aye, that's a good idea.
\end{tabular}

Learning operator.

ExAmple 2-18. The knowledge is acquire but without transformation.

Table A.6 shows an example of knowledge acquisition without transformation in which DB and MH acquired a piece of knowledge from GM directly.

Example 2-19. Discovery is identified as a knowledge transformer.

In Table A.14, discovery was identified as a knowledge transformer in which GM discovered that MH's design idea was not feasible because he forgot the " $Z$ direction."

Example 2-20. Derivation is identified as a knowledge transformer.

Table A.15 illustrates an example of derivation identified as a knowledge transformer in which GM derived the knowledge from $\mathrm{PH}$.

ExAmple 2-21. Specialization is identified as a knowledge transformer.

Table A.14. An example of discovery identified as a knowledge transformer

\begin{tabular}{lll}
\hline \hline No. & \multicolumn{1}{c}{ Transcript } \\
\hline $20 \quad$ MH: (drawing) That's the gantry \\
there. You can have it sitting \\
up there \\
$O_{1}(\mathrm{GM} / \mathrm{MH})$ : Discovery
\end{tabular}$\quad$\begin{tabular}{l} 
GM: $\begin{array}{l}\text { No, no, no but re- } \\
\text { member you've got a } \\
Z \text { direction. . }\end{array}$ \\
\hline
\end{tabular}

Table A.15. An example of derivation identified as a knowledge transformer

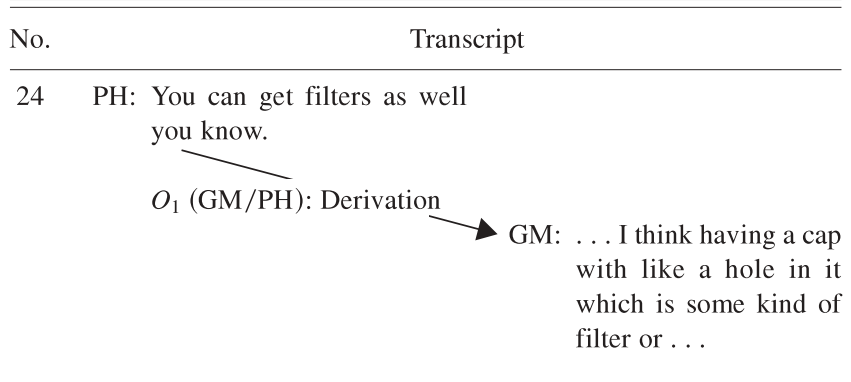

In the learning activity in Table A.16, specialization was identified as a knowledge transformer in that CF learned the meaning of heating unit from $\mathrm{MH}$ and applied it to evaluate the heating elements in their design.

\section{A.2.3. Forms}

Forms with respect to agent interactions.

ExAmPLE 2-22. One-one learning was identified.

Table A.1 illustrates an example of one-one learning in which $\mathrm{MH}$ learned a piece of knowledge from GM that the cartridge should not be sealed.

EXAMPLE 2-23. Mutual learning was identified.

Table A.12 shows one example of mutual learning in which GM and MH mutually evolved their knowledge of the flow control. The mutual learning process is described as: GM had input knowledge on using computer system to control flow; $\mathrm{MH}$ learned from GM that a computer system could be used to control the flow, and generated the knowledge that the flow could be controlled by soaking the sponge; GM learned MH's knowledge and produced another piece of knowledge that the way of soaking the sponge could have the danger of letting the printer run dry.

Table A.16. An example of specialization identified as a knowledge transformer

\begin{tabular}{l}
\hline No. \\
\hline 2 MM: Aye, well it's the value of \\
how well it $\ldots$ \\
MM: Aye, the higher the value \\
the more. .
\end{tabular}


Table A.17. An example of one-many learning

\begin{tabular}{l}
\hline \hline No. \\
\hline $19 \quad$ TH: I think we should just make the thing as small as possible \\
and have like a screw cap on the top or something to refill. \\
Because there's no point in making it totally massive when \\
somebody could just easily pour more fluid in. \\
.. \\
DB: Aye, but if you just have like a simple ... \\
GM: Aye, just a simple, just a small-scale one. \\
PH: Aye.
\end{tabular}

ExAmple 2-24. One-many learning was identified.

Table A.17 illustrates one example in which DB, GM, and PH learn from $\mathrm{MH}$ that the size of the tank will be designed small.

ExAmple 2-25. Common learning was identified.

In the example in Table A.6, GM learned from Gerry (the client of the design project) that they had to use a cartridge because they are using the cradle, indicated from the key words "Right, we have to use cartridge because we are using the cradle. Because Kevin said so ..." GM shared this piece of knowledge to the rest of the team members. It can be inferred that DB and $\mathrm{MH}$ learned this piece of knowledge through their verbalization "yeah." It can also be inferred that the rest of the members learned this knowledge from the context of the protocol, for in their following conversations they started to discuss how to design the cartridge. Common learning can also be identified by one designer's verbalization. For example, the verbalization "Gerry told us what the maximum volume was for that kind of build ..." indicates that all the team members learned the same piece of knowledge, the maximum volume, from Gerry.

Example 2-26. Many-one learning was identified.

In the example in Table A.18, GM learned from Ryan and Paul who had similar ideas to use a syringe to control the flow, indicating from GM's verbalization "can we not have one of those remote control syringe things that Ryan was talking about?" and "Paul sketched one the other day and it is just like a rack and pinion type thing." Many other agents and the agent himself/herself can pro-

Table A.18. An example of many-one learning

\begin{tabular}{l}
\hline No. \\
\hline $10 \quad$ GM: We can do that, but we still have to decide how to meter off \\
the flow at the top end. That's gonna' be like a syringe or \\
something ... Can we not have one of those remote control \\
syringe things that Ryan was talking about? You control \\
medical doses by having a gradual plunger thing, (draws) \\
designed to steadily go down. Paul sketched one the other \\
day and it is just like a rack and pinion type thing. \\
(Explains diagram ....)
\end{tabular}

vide input knowledge for a learning activity. For example, Table A.2 illustrates an example in which GM combined her own and $\mathrm{PH}$ and MH's knowledge. The knowledge that a cap with filters was used to prevent the contamination is produced.

EXAMPLE 2-27. Many-many learning was identified.

In the two meeting sessions of team two, there were indications that members in the team learned from different people in the client company, that was an example of many-many learning. For example, in NB's verbalization, "we were speaking to Malcolm (one of staff in the client company) and he was talking about how you want to make the outside of the tank as narrow as possible for transportation but you've got to maintain your internal diameter for the volume. Will we just start with this 9-m high tank and 3-m diameter," it is indicated that the team learned from Malcolm. In another example, MM had a phone conversation with Alan (another staff in the client company), regarding these questions, indicated from these key words "I'll just start off-the first question was relating to the fill-pipe you have with your tanks" and "The second question is about standard tank volumes." He shared the learned knowledge gained from the client with the rest of the team, indicated from his key words "I'll just tell you what he said actually: he says it can be inside or outside depending on what the customers say," "he says to kind of base it on $50,60,80$, or 100 tonne tanks; that's the standard volume of tanks that they work with and use that to base the standard heights or whatever," and "He says the maximum outside diameter of the thing could be like $3.64 \mathrm{~m}$." As a result, others learned from Alan indirectly.

Example 2-28. One-one-many learning was identified.

Table A.6 illustrates an example of one-one-many learning. GM learned a piece of knowledge from Kevin and then shared that knowledge with other designers. As a result, the rest of the team members learned that knowledge.

Forms with respect to goal of learning.

EXAmple 2-29. Assisted learning was identified.

Table A.10 depicts an example of learning aiming to improve one agent. In this example, PH assisted GM to learn the design idea of the cartridge with his explanation. Table 6 shows an example in which both $\mathrm{MH}$ and $\mathrm{PH}$ assisted GM to learn.

EXAMPLE 2-30. Joint learning was identified.

In the design process of team two, examples are observed that they assisted one another to learn the diagrams of the past designs. Table A. 4 depicts KS, MM, and CF assisting one another to learn the layout of the heating elements.

Forms with respect to an agent's involvement.

EXAMPLE 2-31. Not necessary to be involved learning was identified.

In Table 6, two designers, $\mathrm{MM}$ and $\mathrm{PH}$, provided alternative ways that could be used to control the flow. Thus, the involvement of MM or PH was not a necessary condition for GM's learning activity. 
ExAmple 2-32. Must be involved learning was identified.

Table A.19 shows such an example in which the designers in the team did not know whether the fill-pipe was inside the aluminium skin and what the size of the standard tank was, and they phoned their client (i.e., Alan or Malcolm) to get the answers.

Table A.19. An example of the involvement of an agent being a necessary condition for learning

\begin{tabular}{ll}
\hline \hline No. & \multicolumn{1}{c}{ Transcript } \\
\hline 23 & MH: (this is a telephone conversation) ... We're having a group \\
& meeting just now and we've got a few questions we wanted \\
& to ask either yourself (Alan) or Malcolm ... \\
& I'll just start off—-the first question was relating to the \\
& fill-pipe you have with your tanks. \\
& .. \\
& The second question is about standard tank volumes ... \\
&
\end{tabular}

Thus, the involvement of Alan or Malcolm was a necessary condition to the learning activity.

\section{A.2.4. Collective memory}

EXAmple 2-33. What is learned is used for future design practice.

Table A.18 illustrates an example that GM learned the design idea of the way to control flow from Ryan and Paul and used that knowledge in the existing design.

ExAmple 2-34. What is learned is used for current design practice.

Table A.16 shows an example that CF learned the meaning of the heating unit and applied that knowledge during the design process.

EXAMPLE 2-35. What is learned is used for current design/ learning activity.

Table A.4 illustrates an example that CF learned from the client and shared that learned knowledge for their current learning activity. 